\title{
Muramyl dipeptide activation of nucleotide- binding oligomerization domain 2 protects mice from experimental colitis
}

\author{
Tomohiro Watanabe, ${ }^{1,2}$ Naoki Asano, ${ }^{1}$ Peter J. Murray, ${ }^{3}$ Keiko Ozato, ${ }^{4}$ Prafullakumar Tailor, ${ }^{4}$ \\ Ivan J. Fuss, ${ }^{1}$ Atsushi Kitani, ${ }^{1}$ and Warren Strober ${ }^{1}$ \\ ${ }^{1}$ Mucosal Immunity Section, Laboratory of Host Defenses, National Institute of Allergy and Infectious Diseases, NIH, Bethesda, Maryland, USA. \\ 2Department of Gastroenterology and Hepatology, Kyoto University Graduate School of Medicine, Kyoto, Japan. ${ }^{3}$ Department of Infectious Diseases, \\ St. Jude Children's Research Hospital, Memphis, Tennessee, USA. ${ }^{4}$ Laboratory of Molecular Growth Regulation, \\ National Institute of Child Health and Human Development, NIH, Bethesda, Maryland, USA
}

\begin{abstract}
The mechanisms underlying the susceptibility of individuals with caspase recruitment domain 15 (CARD15) mutations and corresponding abnormalities of nucleotide-binding oligomerization domain 2 (NOD2) protein to Crohn disease are still poorly understood. One possibility is based on previous studies showing that muramyl dipeptide (MDP) activation of NOD2 negatively regulates TLR2 responses and that absence of such regulation leads to heightened Th1 responses. We now report that administration of MDP protects mice from the development of experimental colitis by downregulating multiple TLR responses, not just TLR2. The basis of these in vivo findings was suggested by in vitro studies of DCs, in which we showed that prestimulation of cells with MDP reduces cytokine responses to multiple TLR ligands and this reduction is dependent on enhanced IFN regulatory factor 4 (IRF4) activity. Further studies of mouse models of colitis showed that this inhibitory role of IRF4 does in fact apply to MDP-mediated protection from colitis, since neither IRF4-deficient mice nor mice treated with siRNA specific for IRF4 were protected. These findings indicate that MDP activation of NOD2 regulates innate responses to intestinal microflora by downregulating multiple TLR responses and suggest that the absence of such regulation leads to increased susceptibility to Crohn disease.
\end{abstract}

\section{Introduction}

Nucleotide-binding oligomerization domain 2 (NOD2) is a cytosolic protein that has an essential role in innate immunity as a sensor of a peptide derived from bacterial peptidoglycan (PGN), muramyl dipeptide (MDP) (1). NOD2 consists of a C-terminal leucine-rich repeat region (LRR) for ligand recognition, a central $\mathrm{NOD}$, and $2 \mathrm{~N}$-terminal caspase recruitment domains (CARDs) (1, 2). Upon sensing MDP, NOD2 undergoes conformational changes that lead to a CARD-CARD interaction with RICK, a downstream effector molecule. The latter then ubiquitinates NEMO (IKK- $\gamma$ ) and induces the activation of NF-KB (3).

The functional importance of NOD2 is underscored by the fact that a subpopulation of patients with Crohn disease bears mutations in the CARD15 gene encoding NOD2 $(4,5)$. Such mutations lead to NOD2 molecules that exhibit defective NF-кB activation upon stimulation with MDP (6-8), and thus, at first sight, NOD2 mutations associated with Crohn disease appear to be loss-offunction defects. It should be noted, however, that PGN is recognized independently of its MDP components by cell-surface TLR2

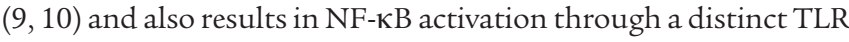

Nonstandard abbreviations used: BMDC, bone marrow-derived DC; CARD, caspase recruitment domain; $\gamma$ DGDAP, $\gamma$-D-glutamyl-diaminopimelic acid; dsRNA, double-stranded RNA; DSS, dextran sodium sulfate; HVJ, hemagglutinating virus of Japan; IRAK-M, IL-1 receptor-associated kinase M; IRF4, IFN regulatory factor 4; LP, lamina propria; LRR, leucine-rich repeat region; MDP, muramyl dipeptide; MLN, mesenteric LN; NLR, nucleotide-binding oligomerization domain-like receptor; NOD2, nucleotide-binding oligomerization domain 2; OT-II, OVA-T cell receptor; $\mathrm{Pam}_{3} \mathrm{CSK} 4, \mathrm{~N}$-palmitoyl(S)-[2,3-bis(palmitoyloxy)-(2Rs)-propyl]Cys-Ser-Lys4; PGN, peptidoglycan; TNBS, trinitrobenzene sulfonic acid.

Conflict of interest: The authors have declared that no conflict of interest exists. Citation for this article: J. Clin. Invest. 118:545-559 (2008). doi:10.1172/JCI33145. signaling pathway involving MyD88. This introduces the possibility that TLR2 and NOD2 signaling are linked and that one function of NOD2 is the regulation of TLR2 signaling. Evidence that this is in fact the case comes from previous findings that show that MDP activation of NOD2 in wild-type murine APCs inhibits PGN-mediated IL-12 production and that NOD2-deficient APCs exhibit greatly enhanced IL-12 production (11). These findings suggest that NOD2 mutations in Crohn disease are, in effect, gainof-function defects with respect to IL-12 production that thus are consistent with the fact that enhanced NF-кB-related Th1 cytokine responses play a critical role in the immunopathogenesis of this disease (12-14). This conclusion is not negated by other data showing that MDP-induced NOD2 signaling may enhance IL-8, TNF, and IL- $1 \beta$ production, usually via TLR pathways (15-17); this latter fact leads to the suggestion that NOD2 can affect TLR-mediated cytokine responses in both a negative and positive fashion under different circumstances.

In a previous study (18), we showed that NOD2-deficient mice adoptively transferred OVA-specific $\mathrm{CD}^{+} \mathrm{T}$ cells and, administered intrarectal Escherichia coli expressing OVA (ECOVA), developed colitis associated with the expansion of OVA-specific $\mathrm{CD}^{+} \mathrm{T}$ cells producing IFN- $\gamma$. Importantly, this colitis was dependent upon TLR2 signaling, since inflammation is suppressed in NOD2 and TLR2double-deficient mice. These data showing that deficient NOD2 signaling predisposed to colitis prompted us to test the possibility that enhanced NOD2 signaling protects mice from the development of colitis. Accordingly, we determined in the present study whether administration of MDP and the attendant increase in NOD2 stimulation could prevent experimental colitis, in this case induced by trinitrobenzene sulfonic acid (TNBS) or dextran sodium 
A

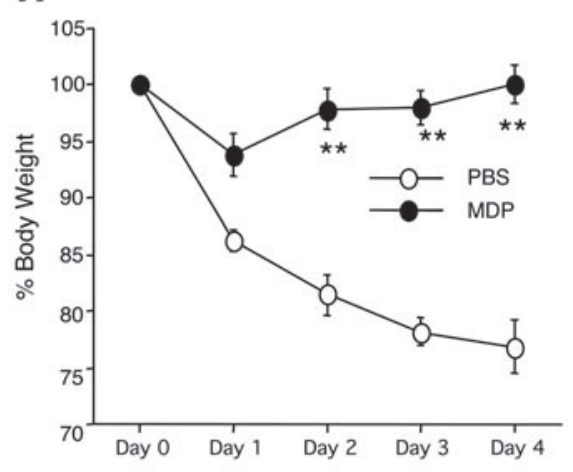

B

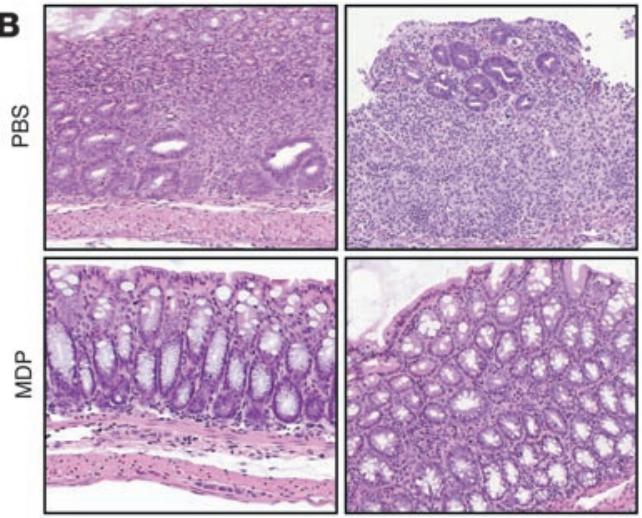

C

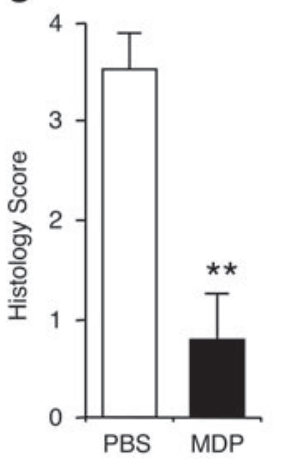

Figure 1

Systemic administration of MDP prevents the development of TNBS colitis. C57BL/10 mice were administered MDP or PBS (i.p.; see Methods) on days $-3,-2$, and -1 and then challenged with intrarectal TNBS on day 0 . (A) Changes in body weight in mice treated with PBS or MDP $(n=10)$ and challenged with intrarectal administration of TNBS. ${ }^{* *} P<0.01$ compared with mice treated with PBS. (B) H\&E-stained colonic tissue of mice harvested on day 4. Histology of PBS-treated mice showed massive infiltration of mononuclear cells as well as destruction of crypt architecture (top row); histology of MDP-treated mice showed almost normal colonic tissue with minimal infiltration of mononuclear cells (bottom row). Original magnification, $\times 100$. (C) Histology score of the colonic tissue of the mice harvested on day 4.

sulfate (DSS). Indeed, we found that pretreatment with MDP completely protected mice from TNBS or DSS colitis and that such prevention was associated with the suppression of multiple TLR signaling pathways, not just TLR2 signaling. In further studies exploring the mechanism of this NOD2-mediated suppression of inflammation, we found that prestimulation of APCs (human DCs) with MDP reduced proinflammatory cytokine production upon subsequent stimulation with TLR2, TLR3, TLR4, TLR5, and TLR9 ligands and that these widespread negative effects of NOD2 signaling were dependent on the induction of a protein that has recently been shown to be a negative regulator of TLR signaling, IFN regulatory factor 4 (IRF4) $(19,20)$. On this basis, we returned to the studies of the protective MDP effects on murine colitis and showed that these effects were also mediated by IRF4.

\section{Results}

Administration of MDP protects mice from TNBS colitis. We previously reported that simultaneous stimulation of murine APCs with PGN and MDP led to a reduction of IL-12 production induced by PGN stimulation of APCs via TLR2 (11) and that this inhibitory effect of NOD2 on TLR2 signaling plays an important role in the prevention of colonic inflammation driven by microbial antigens (18). Furthermore, we recently found that NOD2-transgenic mice expressing NOD2 under the control of class II promoter or administered a plasmid expressing NOD2 manifest decreased susceptibility to TNBS colitis (21). These findings led us in the present study to determine whether administration of MDP protects mice from experimental colitis.

In the first form of experimental colitis we studied, hapteninduced colitis induced by administration of TNBS, C57BL/10 mice were administered MDP (100 $\mu$ g i.p.) or PBS (i.p.) for 3 consecutive days (days -3 to -1 ) prior to intrarectal injection of 3.75 $\mathrm{mg}$ of TNBS in $45 \%$ ethanol (day 0 ). As shown by the body weight curves depicted in Figure 1A, MDP administration prior to the TNBS challenge protected mice from the loss of weight normally seen during the development of TNBS colitis. Moreover, as shown in Figure 1B, whereas mice that were administered PBS prior to intrarectal TNBS instillation exhibited destruction of crypt architecture and infiltration of mononuclear cells in the colonic lamina propria (LP) in tissue examined on day 4 after challenge with TNBS, mice that were administered MDP prior to intrarectal TNBS instillation exhibited little epithelial damage or cellular infiltration. As shown in Figure 1C, this was confirmed by the colitis scores. Thus, these data provide strong evidence that MDP administration prior to TNBS challenge inhibits the development of TNBS colitis.

We next determined whether this prevention of colitis development was associated with reduced inflammatory cytokine responses to microbial antigens, i.e., TLR ligands and nucleotide-binding oligomerization domain-like receptor (NLR) ligands. For this purpose, mesenteric LNs (MLNs) and LP cells isolated from mice pretreated with MDP or PBS before TNBS challenge were stimulated with a broad range of TLR ligands. As shown in Figure 2, A and B, MLN cells and colonic LP cells obtained from mice administered MDP and then stimulated with a broad range of TLR ligands displayed markedly reduced production of IL-12p40, IL-12p70, IL-6, and TNF upon stimulation with TLR2, TLR3, TLR4, TLR5, and TLR9 ligands as compared with cells obtained from mice administered PBS before TNBS challenge. Thus, prior activation of NOD2 by MDP administration caused suppression of multiple TLR pathways, not just TLR2. Furthermore, these reduced innate cytokine responses were associated with reduced adaptive Th1 responses since, as shown in Figure 2C, MLN and colonic LP cells from MDP-treated mice exhibited markedly reduced IFN- $\gamma$ production upon anti-CD3 stimulation. We next determined whether reduced cytokine responses were due to the downregulation of NF- $\mathrm{KB}$ activation. Accordingly, we isolated nuclear proteins from MLN cells and assessed such activation by both EMSA and a semiquantitative method based on the binding of the extract to NF-kB consensus sequences, followed by detection of bound components with subunit-specific Abs (NF-KB ELISA study) (11). As shown in Figure 2, D and E, NF- $\mathrm{KB}$ activation by LPS or PGN stimulation was suppressed in MLN cells from MDP-treated mice as compared with those from PBS-treated mice. Taken together, these data suggest that MDP 
A
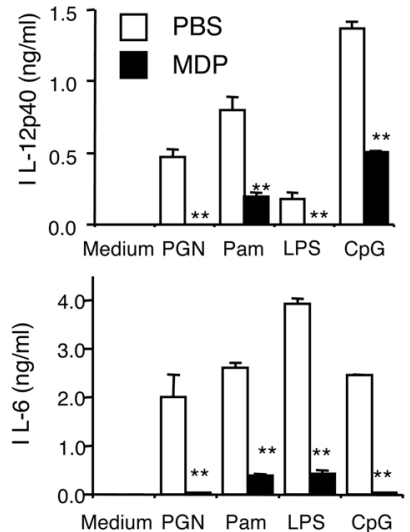

B
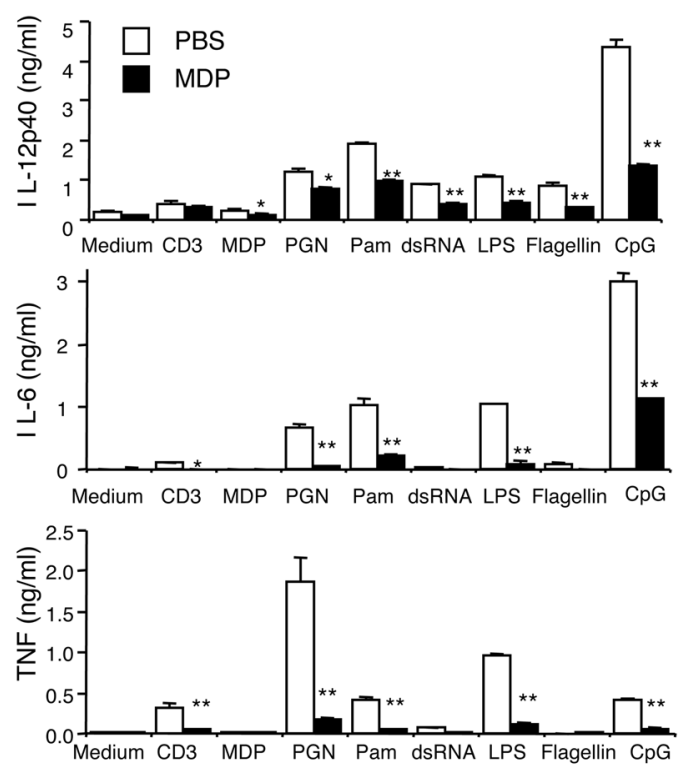
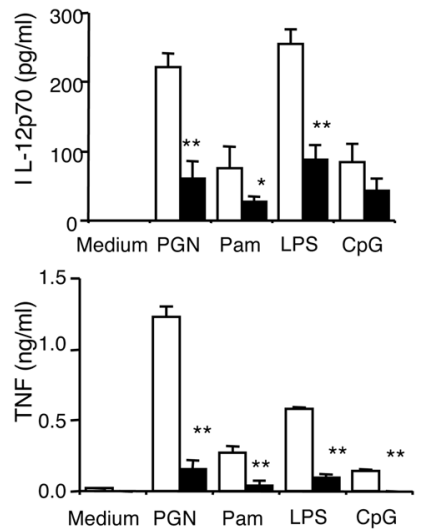

D
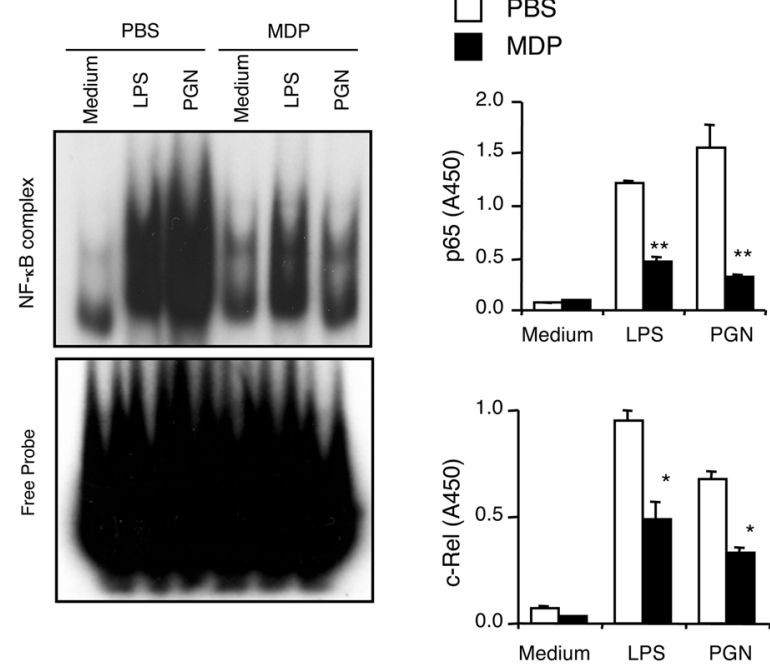

Figure 2

MDP administration reduces the TLR-induced cytokine responses of MLN and colonic LP cells from mice with TNBS colitis. (A) Colon LP lymphocytes $\left(1 \times 10^{6} / \mathrm{ml}\right)$ isolated from the mice on day 3 were stimulated with PGN, Pam ${ }_{3}$ CSK4, LPS, and CpG in the presence of IFN- $\gamma(20$ $\mathrm{ng} / \mathrm{ml}$ ); cultured supernatants were collected at 48 hours and analyzed for cytokine production. ${ }^{*} P<0.05 ;{ }^{* *} P<0.01$ when supernatants from MDP-treated mice were compared with supernatants from PBS-treated mice. (B) MLN cells $(1 \times 10 \% / \mathrm{ml})$ isolated from mice with TNBS colitis on day 3 were stimulated with anti-CD3 $(1 \mu \mathrm{g} / \mathrm{ml})$ and a broad range of TLR ligands. Cultured supernatants were collected at 48 hours and analyzed for cytokine production. ${ }^{*} P<0.05$; ${ }^{* *} P<0.01$ when supernatants of MDP-treated mice were compared with supernatants of PBS-treated mice. (C) MLN cells and colon LP lymphocytes isolated from the mice on day 3 were stimulated with anti-CD3 $(1 \mu \mathrm{g} / \mathrm{ml})$; cultured supernatants were collected at 48 hours and analyzed for IFN- $\gamma$ production. ${ }^{* *} P<0.01$ when supernatants from MDP-treated mice were compared with supernatants from PBS-treated mice. (D) Evaluation of NF-KB activation in MLN cells isolated from the mice on day 3 and stimulated with LPS or PGN. Nuclear extracts were prepared from MLN cells isolated from PBS- or MDP-treated mice and stimulated with LPS or PGN for 2 hours and then subjected to EMSA. (E) Nuclear extracts obtained in $\mathbf{D}$ assayed for $\mathrm{p} 65$ and c-Rel activation using NF-KB transcription factor ELISA. ${ }^{*} P<0.05$; ${ }^{\star *} P<0.01$ compared with nuclear extracts from PBS-treated mice.

treatment protects against TNBS colitis by inhibiting responses to a wide range of TLR ligands.

Administration of MDP protects mice from DSS colitis. In studies of a second experimental colitis, DSS colitis, control NOD2-intact or NOD2-deficient mice treated with $4 \%$ DSS in the drinking water from day 0 to day 5 to induce colitis were administered either MDP (100 $\mu$ g i.p.) or PBS (i.p.) for 3 consecutive days at the early phase of the colitis (days $0,1,2$ ). As shown in Figure 3A, NOD2-intact mice with DSS colitis treated with PBS but not those treated with MDP exhibited significant body weight loss during the observation period. Furthermore, this protective effect was mediated by NOD2 activa- tion, since NOD2-deficient mice with DSS colitis exhibited comparable body weight loss whether they were treated with PBS or MDP. As shown in Figure 3B, these body weight data correlated with serum amyloid A levels as well as with the colitis scores of the NOD2-intact mice treated with MDP and PBS, and again, no difference was seen in these parameters in NOD2-deficient mice treated with MDP or PBS. Thus, these studies provide strong evidence supporting the view that MDP administration protects mice from the development of DSS colitis and complement the data above on TNBS colitis.

In studies of the mechanism of such protection, we analyzed the profiles of cytokine production of mice described above that 
A

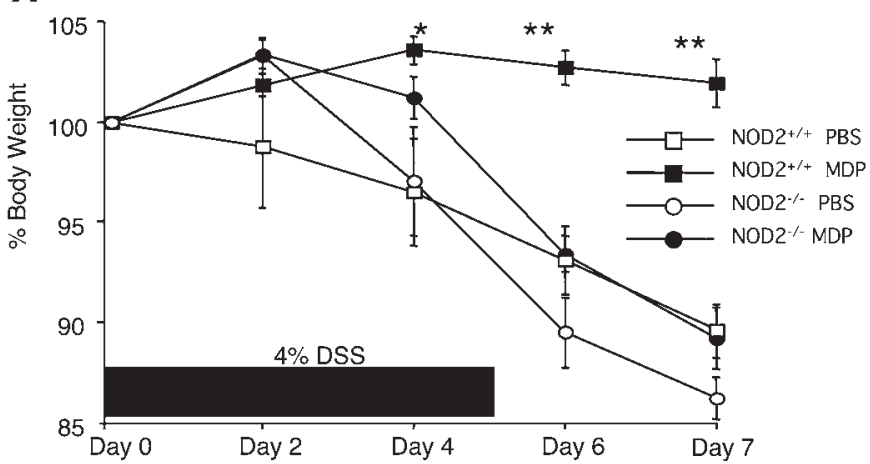

B

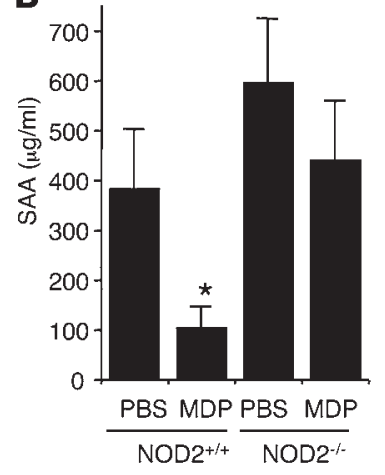

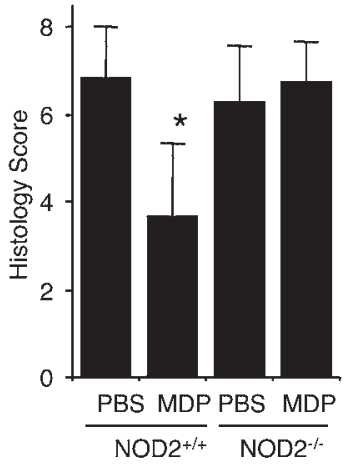

Figure 3

MDP administration prevents DSS colitis. NOD2-intact (NOD2 ${ }^{+/+}$) and NOD2-deficient (NOD2-/-) mice were treated with drinking water containing 4\% DSS for 6 days (days 0-5). At the early phase of colitis induction (days 0, 1, 2), mice were administered MDP (i.p.) or PBS every day. (A) Changes of body weight in PBS- or MDP-injected mice. ${ }^{*} P<0.05 ;{ }^{*} P<0.01$, time point values of NOD2-intact mice administered MDP compared with NOD2-intact mice administered PBS. (B) Serum amyloid A (SAA) levels and colitis scores of mice (see Methods) on day 7. SAA levels were determined by ELISA. ${ }^{\star} P<0.05$, NOD2-intact mice administered MDP compared with mice administered PBS.

received MDP administration at the onset of DSS challenge. For this purpose, MLN cells obtained from mice on day 5 after the initial challenge with DSS were stimulated with several TLR ligands. As shown in Figure 4A, MDP administration was associated with a reduction in TLR2-, TLR3-, and TLR4-mediated IL-12p40 and IFN- $\gamma$ production by MLN cells obtained from NOD2-intact but not NOD2-deficient mice. However, this reduction of IL-12p40 or IFN- $\gamma$ production seen in NOD2-intact mice treated with MDP was not observed in MLN cells from NOD2-deficient mice treated with MDP. Finally, as shown in EMSA and NF-кB ELISA studies in Figure 4, B and C, respectively, PGN or LPS-mediated NF- $\mathrm{B}$ activation was greatly suppressed in MLN cells from NOD2-intact mice treated with MDP as compared with those not so treated. Thus, as in the case of TNBS colitis, MDP pretreatment protects mice from DSS-induced colitis through downregulation of proinflammatory cytokine responses evoked by a number of TLR ligands, not just TLR2.

MDP administration does not protect from DSS colitis in mice with abnormal NOD2 arising from a CARD15 frame-shift mutation. The data above show that MDP activation of NOD2 protects mice from TNBS or DSS colitis by downregulating multiple TLR pathways. To relate these findings to Crohn disease, we next determined whether NOD2 arising from a CARD15 mutation associated with Crohn disease has a similar protective function. To this end, we determined the ability of MDP to protect NOD2-deficient mice reconstituted with plasmids expressing murine intact CARD15, frame-shift CARD15 (L980fs) equivalent to human 3020insC, or control "empty" plasmid from the development of DSS colitis. Accordingly, NOD2-deficient mice were treated with 5.5\% DSS in drinking water from day 0 to day 5 to induce DSS colitis. As in previous studies, the mice were administered MDP (100 $\mu$ g i.p.) for 3 consecutive days beginning at the time of colitis induction (days $0,1,2$ ), but in this case each MDP dose was accompanied by i.p. administration of either intact CARD15, frameshift CARD15, or control empty vector encapsulated in hemagglutinating virus of Japan (HVJ) for efficient in vivo delivery (21). As shown by the weight curves in Figure 5A and the histologic data in Figure 5B, while MDP injection protected NOD2-deficient mice reconstituted with intact CARD15 plasmid, it did not protect NOD2-deficient mice reconstituted with frame-shift CARD15 plasmid or with control empty plasmid. Colitis scores correlated with the tissue histology shown in Figure 5B (data not shown). Thus, these data suggest that abnormal NOD2 arising from the Crohn disease-associated CARD15 frame-shift mutation lacks the ability to control colonic inflammation upon systemic administration of MDP.

Human DCs subjected to preactivation of NOD2 by MDP exhibit reduced proinflammatory cytokine production upon subsequent stimulation with TLR ligands. Since MDP administration inhibits the inflammation occurring in murine models of colitis, it was of interest to determine the conditions under which MDP stimulation could also inhibit inflammatory cytokine responses to multiple TLR ligands in vitro.

In the relevant studies, we determined the effect of MDP prestimulation of cells on the assumption that such prestimulation recapitulated the pretreatment of mice in the above colitis models. In initial studies, we preincubated human monocyte-derived DCs with medium (absence of NOD2 prestimulation) or with MDP (presence of NOD2 prestimulation) for 24 hours prior to stimulation with TLR ligands alone or stimulation with TLR ligands plus MDP (MDP costimulation). As shown in Figure 6A, in keeping with prior results, in the absence of NOD2 prestimulation, PGNmediated IL-12p40 and IL- 6 production was inhibited by NOD2 costimulation, whereas double-stranded RNA (dsRNA), LPS, or CPG-mediated IL-12p40, IL-6, and TNF production was variably enhanced by NOD2 costimulation. In contrast, in the presence of MDP prestimulation, production of proinflammatory cytokines and chemokines such as IL-12p40, IL-6, and CXCL10 stimulated with a wide range of TLR ligands was inhibited and there was reversal of enhancement by NOD2 costimulation. The effect of NOD2 prestimulation and costimulation on TNF production was somewhat different, since here NOD2 costimulation in the absence of NOD2 prestimulation was either associated with no inhibition or enhancement of TLR stimulation; nevertheless, in this case as well, such enhancement was usually reversed by NOD2 prestimulation. Thus, consistent with results obtained from studies of in vivo colitis models, MDP pretreatment has a remarkable inhibitory effect on multiple types of TLR responses.

It is unlikely that the above inhibition of TLR responses by NOD2 prestimulation is due to induction of either cell "exhaus- 
A
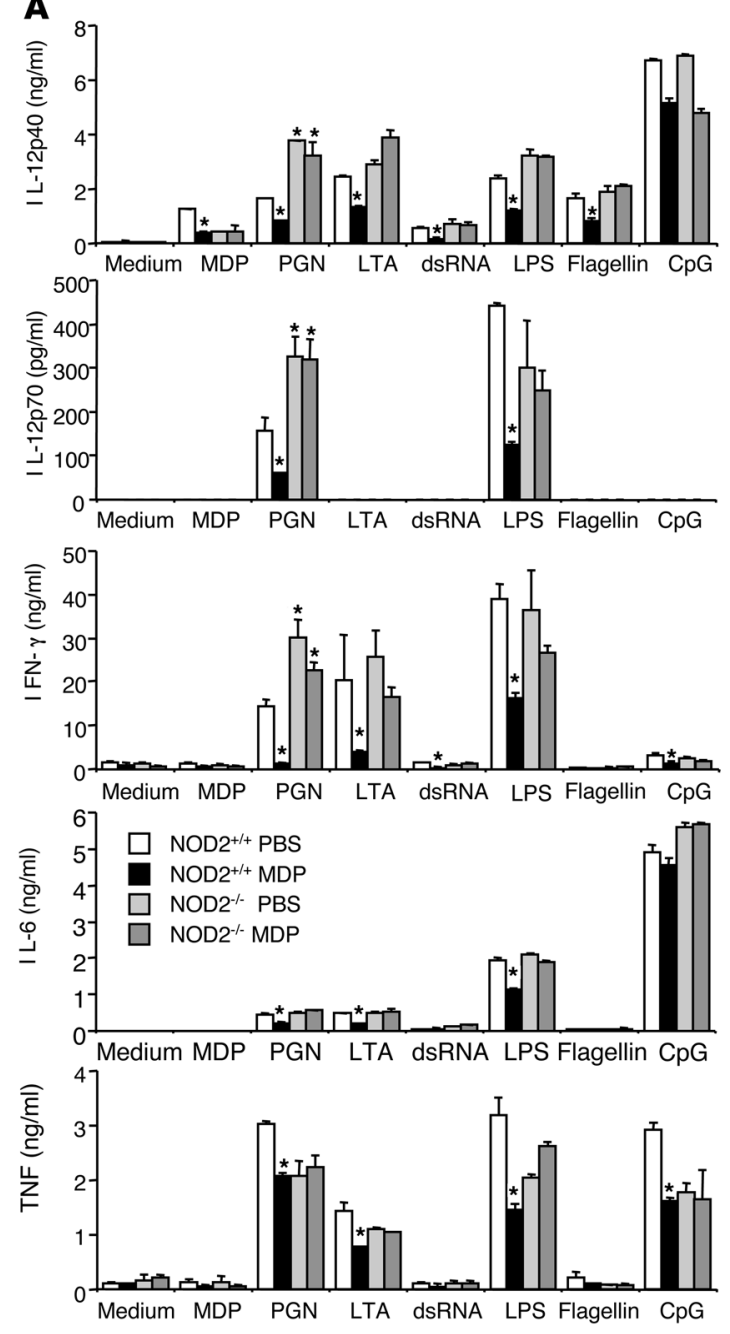

B
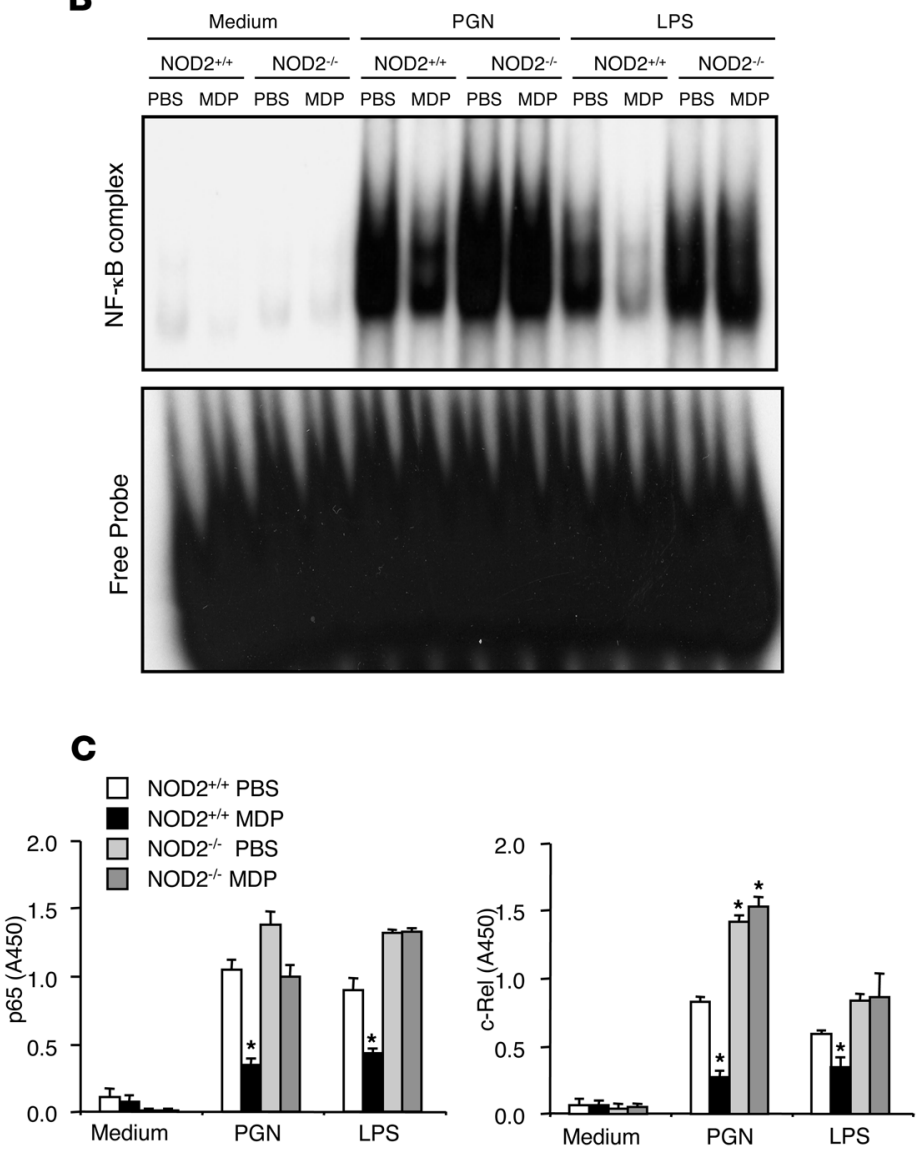

Figure 4

Cytokine production by MLN cells in mice treated with DSS. (A) MLN cells $\left(1 \times 10^{6} / \mathrm{ml}\right)$ isolated from NOD2 $2^{+/}$and NOD2 $2^{-/-}$mice on day 5 were stimulated with a broad range of TLR ligands. Cultured supernatants were collected at 48 hours and analyzed for cytokine production by ELISA. ${ }^{\star} P<0.05$ when supernatants were compared with supernatants from mice treated with PBS (white bars). (B) Activation of NF- $\kappa B$ in MLN cells isolated from $\mathrm{NOD}^{+/+}$and $\mathrm{NOD} 2^{-/-}$mice on day 5 following stimulation with LPS or PGN. Nuclear extracts were prepared from MLN cells isolated from PBS- or MDP-treated mice and stimulated with LPS or PGN for 2 hours and then subjected to EMSA. (C) Nuclear extracts obtained in B assayed for p65 and c-Rel activation using NF-KB transcription factor ELISA. ${ }^{*} P<0.05$ compared with nuclear extracts from PBS-treated mice.

tion" as defined previously (22) or simply to cell death. Thus, as shown in Figure 6A, NOD2 pretreatment had no inhibitory effect on either PGN- or LPS-induced TNF production and, as shown in Supplemental Figure 1A (supplemental material available online with this article; doi:10.1172/JCI33145DS1), no effect on CXCL8 (IL-8) production. In addition, as shown in Supplemental Figure $1 \mathrm{~B}$, pretreatment had no effect on CD40 ligand-induced IL-12p40 or IL-6 production. As shown in Supplemental Figure 1C, pretreatment led to enhanced expression of cell-activation markers without a marked increase in apoptotic cells. Finally, this reduction of proinflammatory cytokine production by NOD2 prestimulation was unlikely to be due to counterregulation by IL-10 since, as shown in Supplemental Figure 1A, IL-10 production was also reduced in DCs subjected to NOD2 prestimulation.

Taken together, these data show that NOD2 prestimulation reduces subsequent TLR-mediated induction of proinflammatory cytokines and chemokines even in situations where simultaneous TLR and NOD2 stimulation are associated with enhancing effects of NOD2 costimulation. In further proof of this conclusion, as shown in Supplemental Figure 2, DCs stimulated by killed E. coli organisms and by multiple TLR ligands simultaneously also exhibited reduced IL-12p40 responses upon NOD2 prestimulation.

Murine bone marrow-derived DCs also exhibit reduced TLR-mediated cytokine responses upon NOD2 preactivation. To both confirm and expand the above findings, we next determined the effect of NOD2 prestimulation on bone marrow-derived DCs (BMDCs) generated from NOD2-deficient mice. In these studies, BMDCs preincubated with MDP for 24 hours were subjected to stimulation with a broad range of TLR ligands. As shown in Figure 6B, NOD2 prestimulation led to a substantial reduction of IL-12p40 and IL- 6 production by NOD2-intact BMDCs stimulated with TLR2, TLR4, TLR5, and TLR9 ligands. In contrast, this inhibitory 
A

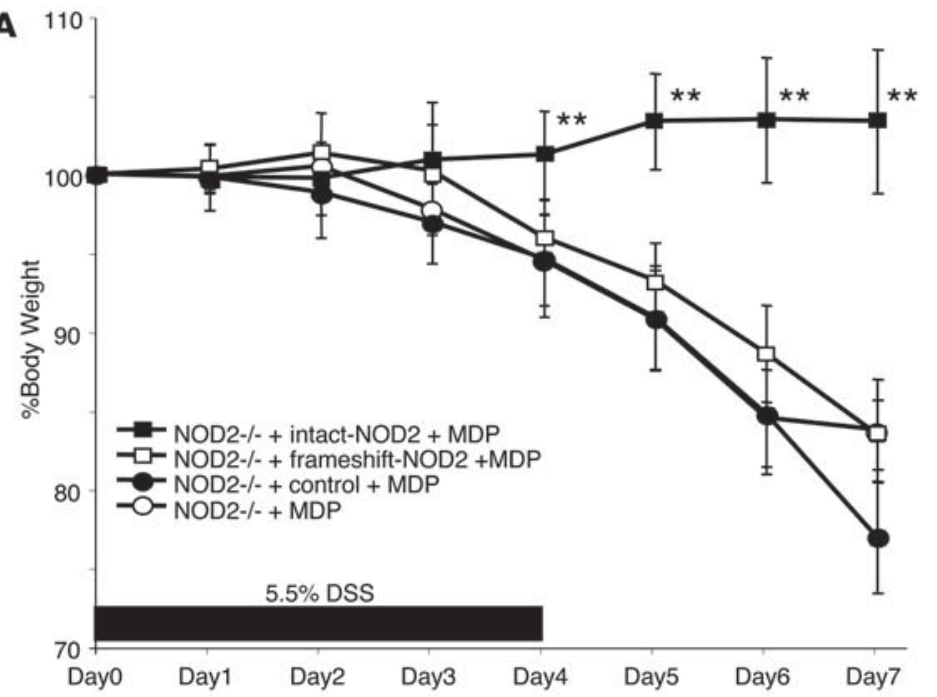

B

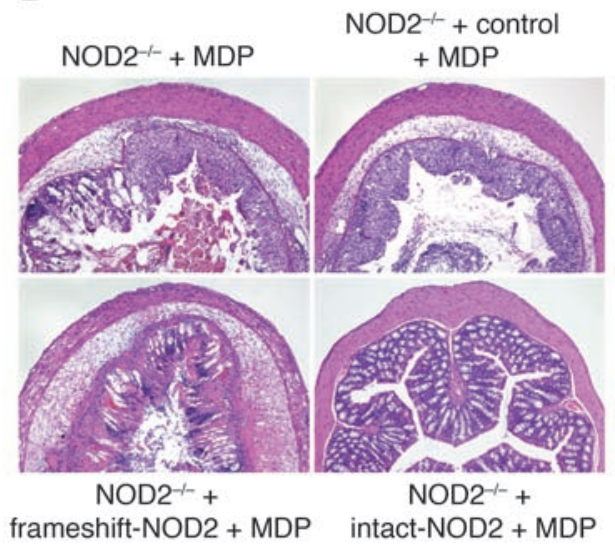

Figure 5

DSS colitis in MDP-treated NOD2-deficient mice reconstituted with intact or frameshift NOD2. NOD2-deficient (NOD2 $\left.{ }^{--}\right)$mice were treated with drinking water containing 5.5\% DSS for 6 days (days $0-5$ ). At an early phase of colitis induction (days $0,1,2$ ), mice were administered MDP and HVJ-encapsulated plasmid (see Methods). (A) Changes in body weight in MDP-administered NOD2-deficient mice reconstituted with intact NOD2, frameshift NOD2, or control empty vector. Weights of MDP-administered NOD2-deficient mice given DSS are shown as a control. ${ }^{\star \star} P<0.01$, time point values of intact-NOD2-reconstituted mice compared with control empty vector-reconstituted mice. (B) H\&E-stained colonic tissue of the mice harvested on day 7 . Original magnification, $\times 50$.

effect was not observed with NOD2-deficient BMDCs. As shown in Supplemental Figure 3A, a similar effect of MDP pretreatment was observed with IL-12p70 and TNF production. Finally, as shown in Supplemental Figure 3B, MDP stimulation did not affect BMDC expression of costimulatory molecules or MHC class II expression. Similarly, MDP treatment did not change BMDC expression of TLR2 and TLR4 (data not shown).

In further studies, we asked whether this reduction of proinflammatory cytokine responses by NOD2 prestimulation results in decreased cytokine responses by naive OVA-specific $\mathrm{CD}^{+} \mathrm{T}$ cells. For this purpose, we determined the ability of DCs subjected to NOD2 prestimulation to induce OVA-specific T cells to produce IFN- $\gamma$ upon coculture with OVA peptide and naive T cells from OVA-T cell receptor (OT-II) transgenic mice. As shown in Figure 6C, OVA peptide presentation by NOD2-intact NOD2-prestimulated BMDCs as compared with nonprestimulated BMDCs led to greatly reduced IFN- $\gamma$ production by $\mathrm{CD}^{+} \mathrm{T}$ cells isolated from the spleens of OT-II transgenic mice in the presence of TLR2, TLR4, TLR5, and TLR9 ligands. In contrast, this reduced response was not observed with MDP-prestimulated NOD2-deficient BMDCs. These studies of mouse DCs thus confirm the inhibitory effect of NOD2 prestimulation observed with human DCs and provide evidence that the effect is in fact mediated by NOD2.

Inbibition of TLR cytokine responses by NOD2 prestimulation is associated with upregulation of IRF4. The fact that we could inhibit multiple TLR responses with NOD2 prestimulation allowed us to explore the molecular basis of the inhibitory effect. Initially, we assessed the effect of MDP prestimulation on subsequent TLR activation of NF- $\kappa \mathrm{B}$ in human DCs by performing gel-shift assays on nuclear extracts isolated from the stimulated DCs. As shown in Figure 7A, nuclear extracts isolated from MDP-prestimulated DCs subsequently stimulated with LPS, PGN, or flagellin gave rise to bands of reduced intensity when incubated with ${ }^{32} \mathrm{P}-$ labeled oligo probes

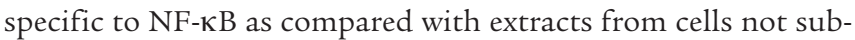
jected to prestimulation. These data suggest that NOD2 prestimulation reduces subsequent activation of NF- $\kappa \mathrm{B}$ by TLR ligands.

In further studies, we performed immunoblot analyses to determine the expression of signaling molecules involved in the TLR signaling pathway or those previously shown to be negative regulators of that pathway $(19,20,23)$ in cells incubated in medium versus cells incubated with MDP or LPS. Because of the results of the gel-shift assay described above, we focused on those molecules involved in the activation of NF- $\mathrm{KB}$. As shown in Figure $7 \mathrm{~B}$, there was no difference in the expression of TLR signaling molecules such as MyD88, TAK1, TRAF6, IRF3, IRF5, or IKK- $\gamma$ in cells without and with either NOD2 (MDP) or TLR4 (LPS) stimulation. In contrast, MDP-stimulated cells expressed increased amounts of the negative regulator IRF4 at 24 hours after stimulation (and, as shown in Supplemental Figure 4A, at 36 hours as well) but not other negative regulators such as IL-1 receptor-associated kinase $\mathrm{M}$ (IRAK-M) or SOCS1. Consistent with previous reports $(19,20,23$, 24), cells stimulated by LPS expressed increased amounts of both IRF4 and IRAK-M but not SOCS1.

Inbibition of TLR cytokine responses by NOD2 prestimulation requires the expression of IRF4. To determine whether NOD2 prestimulation is not only associated with IRF4 expression but actually requires IRF4 expression, we initially asked whether gene silencing of IRF4 or IRAK-M expression by siRNA specific for these molecules affects NOD2- or TLR4-mediated inhibitory effects. As shown in Figure 7C, transfection of human DCs with a mixture of IRF4 siRNA substantially reduced expression of IRF4 at the protein level in MDPand LPS-stimulated human DCs. Similarly, transfection of IRAK-M siRNA reduced expression of IRAK-M in LPS-stimulated cells. As shown in Figure 7D, transfection of IRF4 siRNA but not IRAK-M siRNA led to increased IL-12p40 production in MDP-prestimulated DCs subsequently stimulated with PGN, N-palmitoyl(S)-[2,3- 

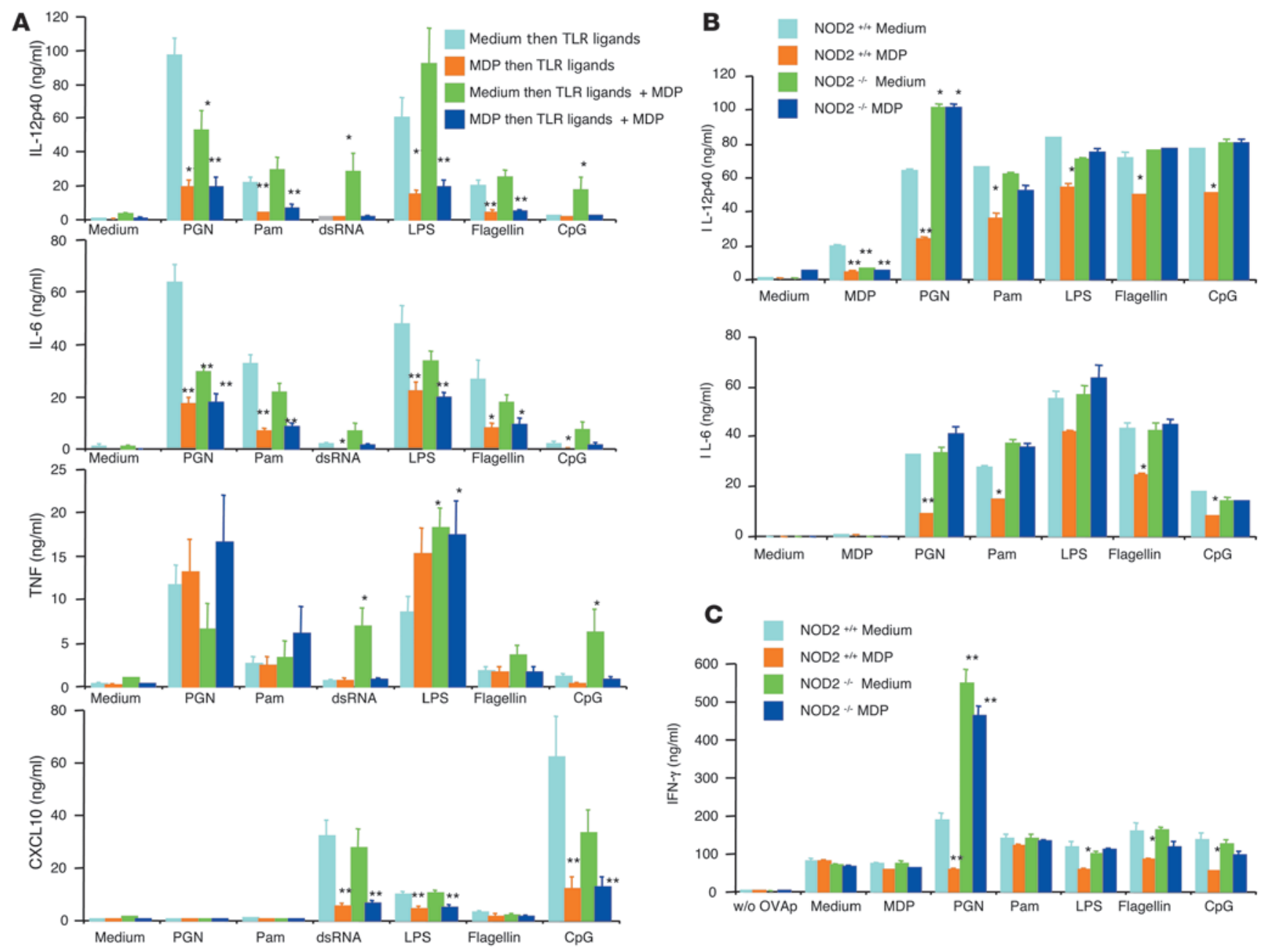

\section{Figure 6}

Human and mouse DCs prestimulated with MDP exhibit reduced cytokine and chemokine production when stimulated with TLR ligands. (A) Human monocyte-derived DCs $(1 \times 10 \% / \mathrm{ml})$ from 6 healthy donors were preincubated with MDP or medium for 24 hours and then stimulated with a broad range of TLR ligands alone or in combination with MDP for an additional 24 hours. Cultured supernatants were collected at 24 hours and analyzed for cytokine and chemokine production by ELISA. ${ }^{*} P<0.05$; ${ }^{* *} P<0.01$ compared with supernatants from DCs preincubated with medium and stimulated with TLR ligands alone (light blue bars). (B) CD11 ${ }^{+}$DCs $\left(1 \times 10^{6} / \mathrm{ml}\right)$ derived from bone marrow cells from NOD2intact (NOD2 ${ }^{+/+}$) and NOD2-deficient (NOD2 $\left.{ }^{-/}\right)$mice were preincubated with MDP $(50 \mu \mathrm{g} / \mathrm{ml})$ or medium alone for 24 hours and stimulated with a broad range of TLR ligands. Cultured supernatants were collected at 24 hours and analyzed for cytokine production by ELISA. ${ }^{\star} P<0.05$; ${ }^{* *} P<0.01$ when supernatants were compared with NOD2-intact DCs preincubated with medium and stimulated with TLR ligands (light blue bars). (C) $\mathrm{OVA}_{323-339}$ peptide-specific CD4+ T cells (OT-II) were purified from the spleens of OT-II transgenic mice; OT-II cells $\left(1 \times 10^{6} / \mathrm{ml}\right)$ were cocultured with NOD2-intact or NOD2-deficient BMDCs $\left(2 \times 10^{6} / \mathrm{ml}\right)$ in the presence of a broad range of TLR ligands and OVA peptide $(0.5 \mu \mathrm{M})$; cultured supernatants were collected at 72 hours and analyzed for IFN- $\gamma$ production by ELISA. ${ }^{*} P<0.05 ;{ }^{* *} P<0.01$ compared with supernatants from NOD2-intact DCs preincubated with medium and stimulated with TLR ligands (light blue bars).

bis(palmitoyloxy)-(2Rs)-propyl]Cys-Ser-Lys4 (Pam ${ }_{3}$ CSK4), and LPS. In addition, as shown in Figure 7D, transfection of IRAK-M siRNA but not IRF4 siRNA led to increased IL-12p40 production in LPS-preincubated DCs stimulated with PGN, Pam ${ }_{3}$ CSK4, and LPS. In a final study along these lines, we determined whether individual IRF4-specific siRNAs could abolish inhibition of NOD2 prestimulation to rule out off-target effects of the siRNA mixture. As seen in the studies shown in Supplemental Figure 4B, addition of 2 different siRNAs again led to loss of the inhibition of NOD2 prestimulation in both PGN- and LPS-stimulated cell cultures.

In a second approach to studying the role of IRF4 in inhibition following NOD2 prestimulation, we examined the capac- ity of such prestimulation to inhibit responses in cells naturally lacking IRF4, such as human monocytes (25). In these studies, we determined whether NOD2 prestimulation of THP1 cells, a monocytic cell line, affects their subsequent TLR-induced cytokine production. As shown previously, THP1 cells express NOD2 $(26,27)$ but, as shown in Figure 8A, do not express IRF4 before or after stimulation by MDP or LPS; in contrast, they express IRAK-M, especially after stimulation with LPS. As shown in Figure 8B, in keeping with the data shown above, MDP prestimulation of THP1 cells did not result in reduced IL-12p40 or TNF responses following subsequent stimulation with PGN, $\mathrm{Pam}_{3} \mathrm{CSK} 4$, or LPS, whereas LPS prestimulation did lead to 
A

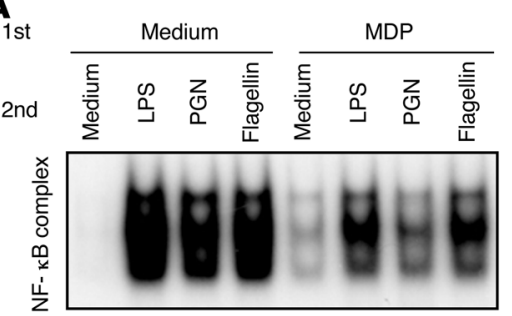

B

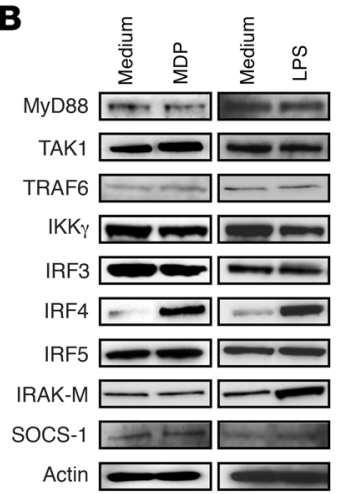

D
C
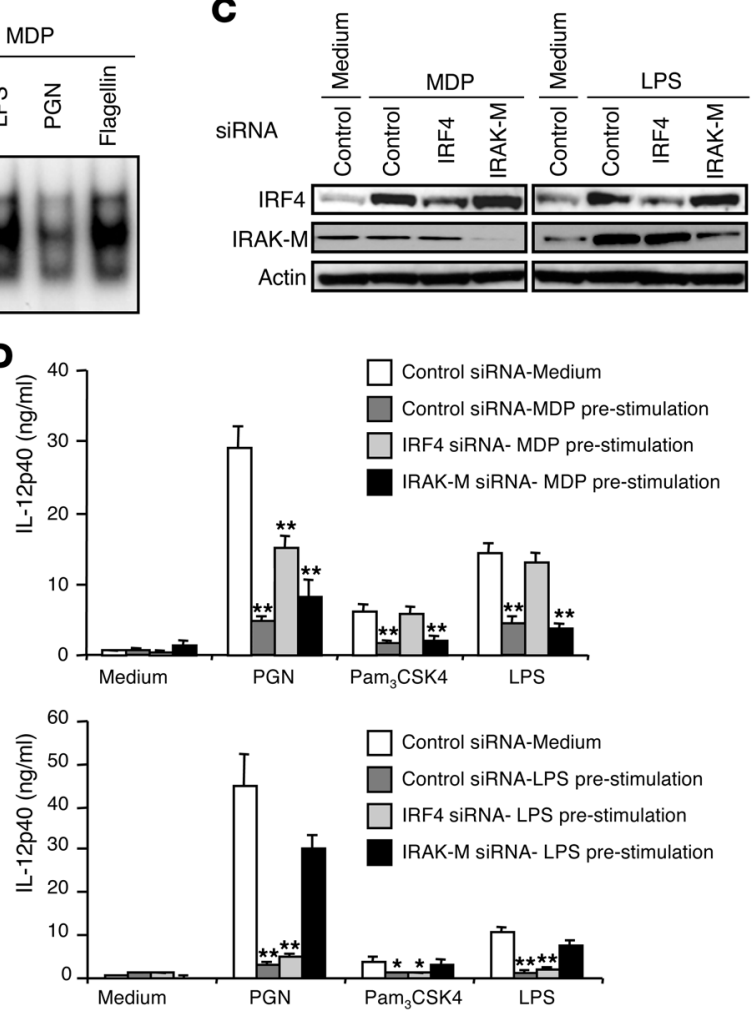

Figure 7

NOD2 stimulation is associated with upregulation of IRF4 expression. (A) NF-kB activation in human monocyte-derived DCs. DCs were preincubated with MDP or medium for 24 hours and then stimulated with LPS, PGN, or flagellin for 2 hours; nuclear extracts of the cells were then obtained and subjected to gel-shift assays; results shown are representative of those obtained with 2 healthy donors. (B) Upregulation of IRF4 in MDP-stimulated human monocyte-derived DCs. Whole-cell extracts prepared from DCs incubated with MDP, LPS, or medium for 24 hours were immunoblotted with Abs against the indicated components; results shown are representative of those obtained in 3 healthy donors. (C) IRF4 expression in monocyte-derived DCs transfected with IRF4 siRNA. DCs were transfected with $2 \mu \mathrm{g}$ of IRF4 siRNA, IRAK-M siRNA, or control siRNA using the Amaxa nucleofection method; 16 hours after transfection, DCs were stimulated with MDP, LPS, or medium for 24 hours, at which point whole-cell extracts were prepared and subjected to immunoblotting with Abs against the indicated components. (D) Effects of IRF4 or IRAK-M siRNA transfection on cytokine production by human monocyte-derived DCs. DCs $\left(2 \times 10^{7} / \mathrm{ml}\right)$ from 6 healthy donors were transfected with IRF4 siRNA, IRAK-M siRNA, or control siRNA, as described in C. After 24 hours of culture with MDP, LPS, or medium, DCs were stimulated with PGN, Pam ${ }_{3}$ CSK4, or LPS for another 24 hours; cultured supernatants were assayed for IL-12p40 by ELISA. ${ }^{*} P<0.05$; ${ }^{*} P<0.01 \mathrm{com}$ pared with DCs transfected with control siRNA and preincubated with medium (white bars).

reduced IL-12p 40 or TNF production by THP1 cells subsequently stimulated with PGN or LPS. Finally, as shown in Supplemental Figure 5, transfection of THP1 cells with an IRF4-expressing vector led to downregulation of PGN, $\mathrm{Pam}_{3} \mathrm{CSK} 4$, and LPS responses, which was particularly evident after MDP prestimulation; thus, the lack of response to MDP prestimulation could in fact be shown to be due to lack of IRF4 expression.

Along similar lines, we determined whether NOD2 prestimulation affected TLR-induced CXCL8 or CXCL10 production in HT-29 cells (i.e., a highly differentiated epithelial cell line), which, like THP1 cells, do not express IRF4. Here again, NOD2 prestimulation did not downregulate responses to subsequent stimulation with dsRNA or flagellin stimulation (data not shown).
The above in vitro studies involving gene silencing of IRF4 and the study of cells naturally lacking IRF4 provide strong support for the view that the inhibitory effects of NOD2 prestimulation depend on IRF4. Further support for this view comes from in vivo studies described below. It should be noted, however, that while IRF4 is necessary for NOD2-mediated inhibition, it is not sufficient for such inhibition. This follows from the fact that, as we have seen, LPS induces IRF4 expression but IRF4 does not mediate LPS-mediated inhibition. A further confirmation of this point came from studies depicted in Supplemental Figure 6A, showing that human DC stimulation with the NOD1 ligand $\gamma$-D-glutamyl-diaminopimelic acid ( $\gamma$ DGDAP) also induced IRF4 expression (albeit less than that induced by the NOD2 ligand), yet, as shown in Supplemental Figure 6B, prestimulation of cells with the NOD1 ligand did not lead to inhibition of subsequent TLR responses.

Sensitivity of PGN and LPS signaling to IRF4 inhibition. The studies described above provide data that suggest that PGN and LPS responses differed in their sensitivity to IRF4-mediated inhibition; NOD2 prestimulation produced more profound inhibition of PGN (TLR2) responses than LPS (TLR4) responses, and suppression of PGN-mediated IL-12p40 production was more resistant to IRF4 siRNA-induced reversal than that of LPS-mediated IL-12p40 production (Figure 7D). To examine this possibility more directly, we measured PGN and LPS responses in human DCs transfected with increasing doses of FLAG-tagged IRF4 cDNA (28). As shown in Supplemental Figure 7, transfection of FLAG-tagged IRF4 induces protein expression in a dose-dependent manner. In addition, as also shown in Supplemental Figure 7, low expression of transfected IRF4 $(0.2 \mu \mathrm{g})$ is sufficient to greatly inhibit PGN-induced production of IL-12p40 and IL-6, whereas in contrast, high expression of IRF4 is necessary to comparably inhibit LPS-induced production of these cytokines. Thus, PGN-mediated TLR2 signaling is more sensitive to negative regulation by IRF4 than LPS-mediated TLR4 signaling. Since IRF4 levels can be assumed to be low in the absence of prestimulation, i.e., when cells are stimulated by TLR ligands and MDP simultaneously, these findings suggest that the reason simultaneous stimulation of TLR ligands and MDP results in reduced PGN-induced production of IL-12p 40 while it has little effect on IL-12p40 production by other TLR ligands is that with simultaneous stimulation the level of IRF4 is sufficient to suppress PGN responses but not responses to other TLR ligands (11). However, further in vivo studies will be necessary to substantiate this possibility. 
A

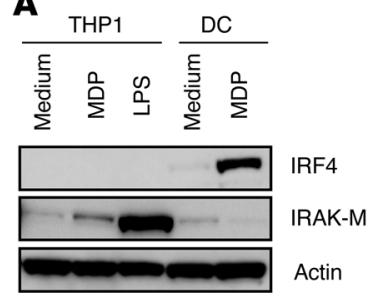

C
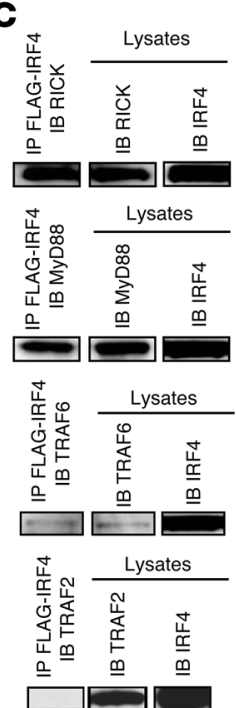

B
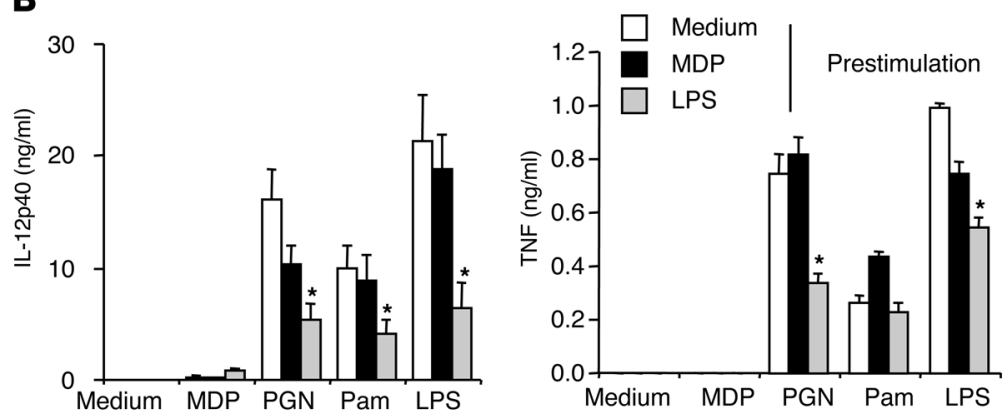

$\mathbf{E}$

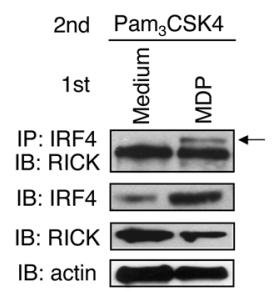

Figure 8

Mechanism of NOD2-induced IRF4 inhibition of TLR signaling. (A) Whole-cell extracts were prepared from THP1 cells stimulated with MDP or LPS for 24 hours and then immunoblotted with Abs against IRF4, IRAK-M, and actin. (B) THP1 cells $(5 \times 105 / \mathrm{ml})$ were prestimulated with MDP, LPS, or medium for 24 hours and stimulated with TLR ligands; cultured supernatants were collected at 24 hours and analyzed for cytokine production by ELISA. ${ }^{*} P<0.05$ compared with the concentrations of cytokines by cells preincubated with medium and stimulated with TLR ligands (white bars). (C) Physical interactions between IRF4 and RICK, MyD88, and TRAF6. Whole-cell extracts of HEK293 cells transfected with vectors (2 $\mu \mathrm{g})$ expressing FLAG-tagged human IRF4 and HA-tagged human MyD88 or with untagged RICK, TRAF6, or TRAF2 were immunoprecipitated with anti-FLAG-conjugated beads and then immunoblotted with anti-HA Abs or with anti-RICK, -TRAF6, or -TRAF2. (D) Negative regulation of NF-KB by IRF4. HT-29 cells $\left(1 \times 10^{5} / 96\right.$-well plate) transfected with pNF-KB-Luc $(50 \mathrm{ng})$ and pSV- $\beta$-galactosidase $(10 \mathrm{ng})$ were cotransfected with vectors expressing human RICK (200 ng), human MyD88 (200 ng), or human TRAF6 (200 ng) with or without an IRF4-expressing vector (50 $\mathrm{ng}, 200 \mathrm{ng}, 1000 \mathrm{ng}$ ). ${ }^{*} P<0.05 ;{ }^{* *} P<0.01$ compared with cells without IRF4 transfection (white bars). (E) Physical interaction of IRF4 and RICK in MDP-prestimulated human DCs. DCs were cultured with MDP or medium for 24 hours and then stimulated with $\mathrm{Pam}_{3} \mathrm{CSK} 4$ for an additional hour; whole-cell extracts were prepared and then immunoprecipitated with anti-IRF4 Abs and immunoblotted with anti-RICK Abs.

Mechanisms of NOD2-induced IRF4 inbibition of TLR signaling. In further studies, we explored the mechanisms underlying NOD2induced inhibition of TLR signaling. In initial studies, we determined possible physical interactions between IRF4 and various components of the TLR signaling pathway. To this end, we performed immunoblots on extracts of HEK293 cells cotransfected with FLAG-tagged human IRF4 cDNA (28) together with either MyD88, TRAF6, or RICK cDNA, all relevant components of the TLR and NOD2 signaling pathways. In addition, we cotransfected the FLAG-tagged human IRF4 cDNA with TRAF2 cDNA, a component of the TNF signaling pathway, as a negative control. As shown in Figure 8C, we found that each of these components except for TRAF2 does indeed bind to IRF4. In further studies, we cotransfected plasmids expressing MyD88, TRAF6, RICK, and IRF4 into HT-29 colon epithelial cells expressing an NF-KB luciferase reporter to determine the capacity of IRF4 to inhibit the capacity of each of the components to activate the reporter and generate luciferase. As shown in Figure 8D, cotransfection of IRF4 led to a dose-dependent reduction in NF- $\kappa B$ activation by each of the components, whereas IRF 4 had no effect on the ability of TNF, a non-TLR/NLR-related NF- $\kappa B$ activator to activate NF-KB. This correlated with the fact that TNF signals mainly through TRAF2, shown above not to interact with IRF4. In additional studies along these lines, we buttressed these overexpression studies with a study of IRF4 binding to RICK in stimulated human DCs (i.e., cells in which IRF4 and signaling components were not overexpressed). As shown in Figure 8E, an immunoblot of extracts of $\mathrm{Pam}_{3}$ CSK4-stimulated cells prestimulated with MDP or medium alone provided evidence that in MDP-prestimulated cells, IRF4 binds to RICK. These data offer evidence that IRF4 inhibition of TLR responses may involve, in part, binding to a key component of the TLR signaling pathway, in line with previous studies of IRF4 inhibition (19, 20). However, further details of the inhibitory mechanism await more complete studies of the functional effects of such binding on NF-KB activation. 

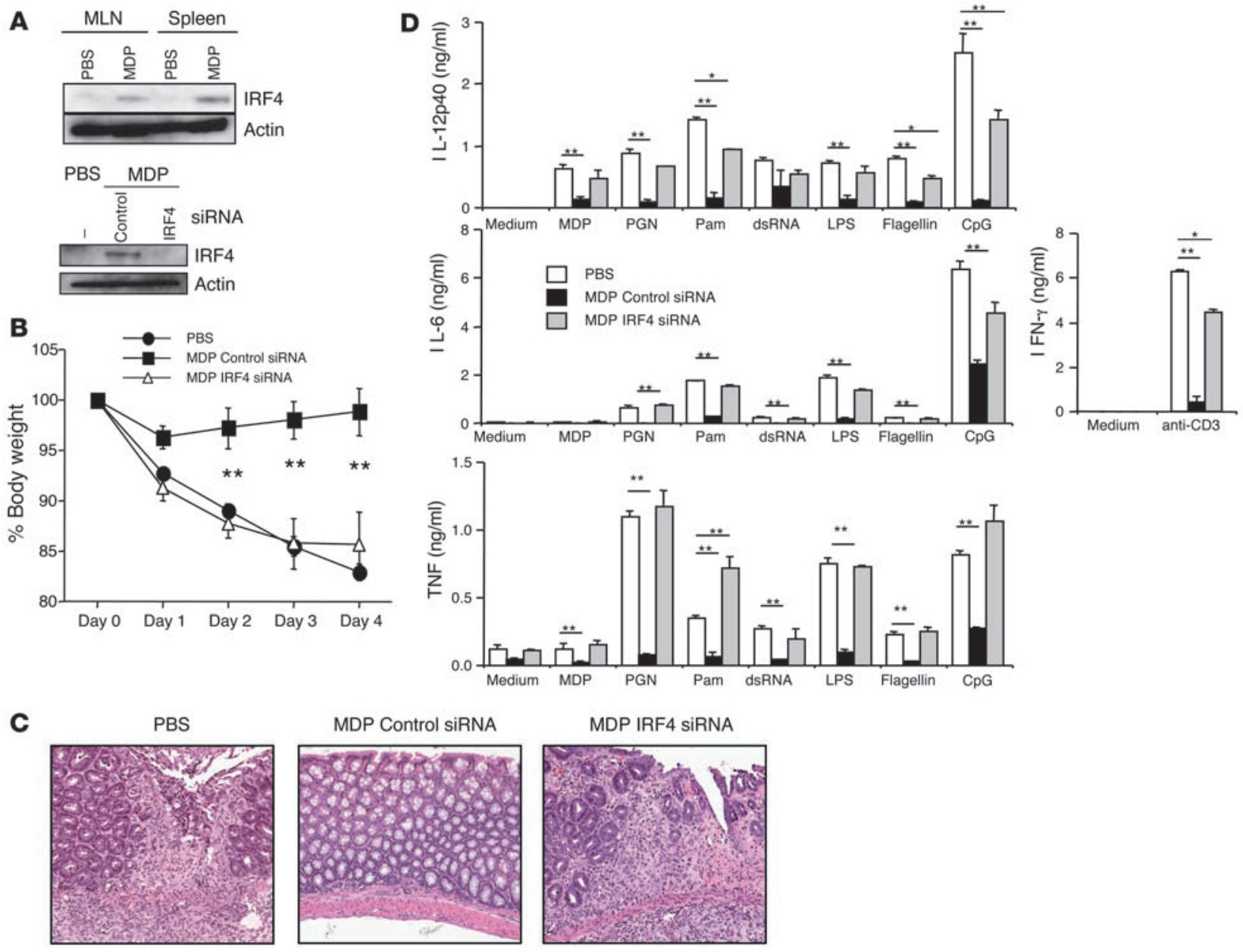

Figure 9

Systemic administration of MDP prevents the development of TNBS colitis by upregulating IRF4 expression. Mice administered intrarectal TNBS on day 0 were injected with MDP or PBS i.p. on days $-3,-2$, and -1 and also administered $100 \mu \mathrm{g}$ of HVJ-encapsulated control siRNA or IRF4 siRNA by intrarectal instillation on days $-2,-1,0$, and 1 . (A) IRF4 expression in CD11 b+ myeloid cells from MLNs and spleens from mice on day 0 (top); IRF4 expression in whole-cell extracts of CD11 b+ myeloid cells from MLNs isolated from mice treated with IRF4 siRNA on day 0 (bottom). (B) Changes of body weight in mice treated with MDP and siRNAs. ${ }^{* *} P<0.01$ compared with body weight of mice treated with PBS. (C) H\&Estained colonic tissue of mice harvested on day 4. Histology of PBS-treated mice and IRF4 siRNA-treated mice showed massive infiltration of mononuclear cells as well as destruction of crypt architecture; histology of control siRNA-treated mice showed almost normal colon tissue with minimal infiltration of mononuclear cells. Original magnification, $\times 100$. (D) MLN cells $(1 \times 106 / \mathrm{ml})$ isolated from mice on day 4 were stimulated with a broad range of TLR ligands; cultured supernatants were collected at 48 hours and analyzed for cytokine production by ELISA. ${ }^{\star} P<0.05$; ${ }^{* \star} P<0.01$ compared with the concentrations of cytokines from PBS-treated mice (white bars).

Systemic administration of MDP prevents the development of TNBS or $D S S$ colitis by upregulating IRF4 expression. The in vitro studies described above provided considerable evidence that the suppression of multiple TLR pathways mediated by MDP activation of NOD2 depends upon the expression of IRF4. To verify this hypothesis in vivo, we returned to the studies of the effect of MDP pretreatment on the development of experimental colitis to determine whether IRF4 also mediated inhibitory MDP effects in these models.

We first asked whether systemic injection of MDP induces upregulation of IRF4 as in the case of human DCs. For this, CD $11 b^{+}$ myeloid cells were isolated from MLNs and spleens of mice that had been administered either MDP or PBS. As shown in Figure 9A, IRF4 protein was barely detectable in cells from PBS-treated mice but was easily detected in cells from MDP-treated mice. We next determined the effect of IRF4 gene silencing on the capacity of MDP administration to protect mice from TNBS colitis. In these studies, mice administered MDP (i.p.) according to the schedule described in Figure 1 were also administered $100 \mu \mathrm{g}$ of either siRNA targeting murine IRF4 or control siRNA encapsulated in HVJ by the intrarectal route on day -2 to day 1 (with respect to TNBS administration). As shown in Figure 9A, induction of IRF4 expression was not seen in CD11b $\mathrm{b}^{+}$MLN cells examined on day 0 obtained from mice administered both MDP and HVJ-IRF4 siRNA, whereas IRF4 expression was clearly seen in cells from mice administered MDP and HVJ-control siRNA. As shown in Figure 9B, while mice administered MDP and control siRNA did not exhibit body weight loss following TNBS challenge, mice administered MDP and IRF4 siRNA exhibited a body weight loss similar to that of mice admin- 

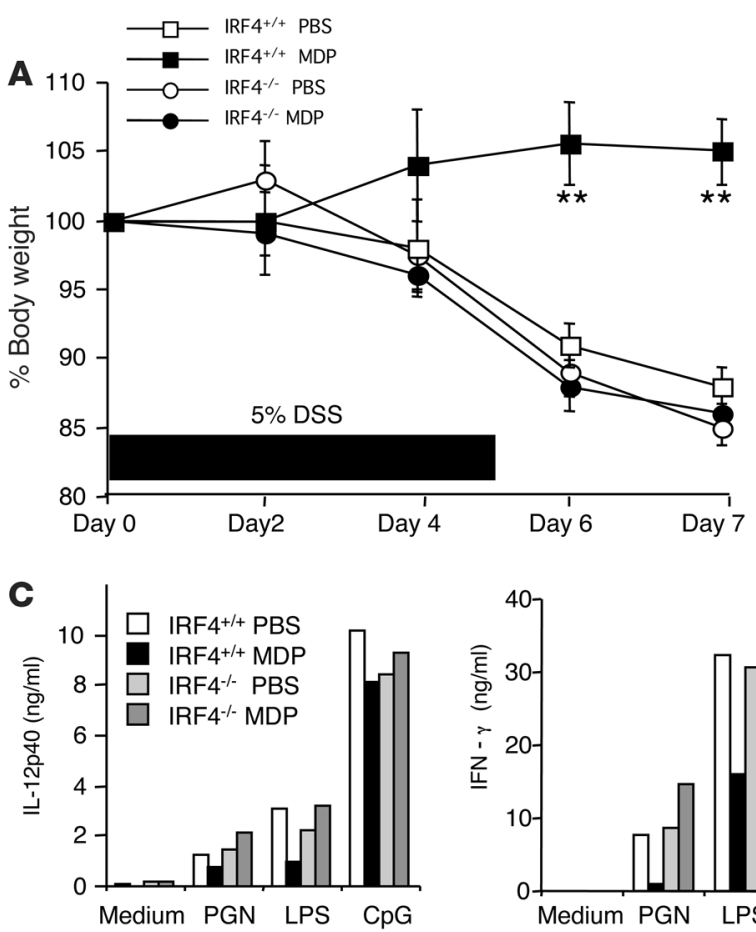

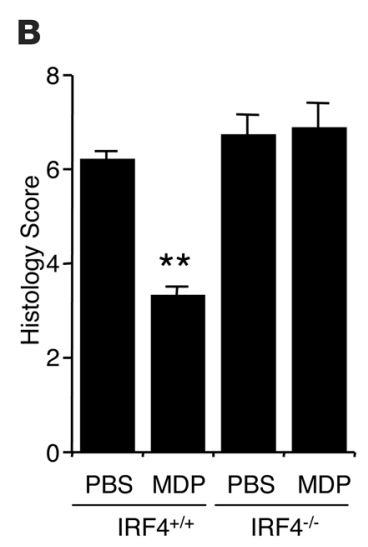

Figure 10

IRF4 signaling is necessary for the suppression of DSS colitis. IRF4-intact (IRF4+/+) and IRF4-deficient (IRF4--) mice were treated with $5 \%$ DSS in drinking water for 6 days (days $0-5$ ). At an early phase of colitis induction (days 0,1,2), mice were administered MDP or PBS (i.p.). (A) Weight curves of $\mathrm{IRF}^{-/-}$or $\mathrm{IRF}^{+/+}$mice administered MDP or PBS. ${ }^{*} P<0.01$ compared with PBS-injected IRF4+/+ mice. (B) Histology score of IRF4-/mice treated with PBS or MDP on day 7. (C) MLN cells $\left(1 \times 10^{6} / \mathrm{ml}\right)$ isolated from IRF4+/+ and IRF4 $4^{-/}$mice on day 7 were stimulated with PGN, LPS, or CpG; cultured supernatants were collected at 48 hours and analyzed for cytokine production by ELISA. istered PBS. As shown in Figure 9C, these weight loss data correlated with pathology: mice administered MDP and IRF4 siRNA exhibited severe colonic epithelial cell damage and massive infiltration of inflammatory cells in the LP equivalent to the changes seen in mice treated with PBS. Finally, as shown in Figure 9D, the weight loss data correlated with cytokine production findings in that the marked reduction in TLR-mediated IL-12p40, IL-6, and TNF accompanying MDP administration was almost completely restored by IRF4 siRNA administration. Taken together, these data show that MDP administration protects mice from TNBS colitis via its ability to induce NOD2 induction of IRF4 and subsequent IRF4 inhibition of TLR-mediated inflammatory cytokine production.

Finally, we asked whether the protection from DSS colitis in mice treated with MDP depends upon IRF4, in this case utilizing a welldescribed gene-targeted mouse with a background strain susceptible to DSS colitis (29). Indeed, immunoblot studies of extracts of spleen cells of IRF4-deficient mice with DSS colitis did not reveal an IRF4 band, whereas IRF4-intact mice did reveal a band (data not shown). As shown in Figure 10A, as judged by body weight loss, MDP administration as described above did not inhibit the development of DSS colitis in IRF4-deficient mice, whereas it did inhibit such colitis in IRF4-intact mice. In addition, as shown in Figure 10B, the colitis scores of MDP-treated and untreated IRF4deficient mice were not significantly different, whereas those of MDP-treated IRF4-intact mice were significantly reduced as compared with untreated IRF4-intact mice. Finally, as shown in Figure 10C, whereas MLN cells from MDP-treated IRF4-intact mice exhibited a clear decrease in both IL-12p40 and IFN- $\gamma$ secretion upon TLR ligand stimulation, comparable cells from IRF4-deficient mice exhibited no such decrease.

\section{Discussion}

In a previous study, we demonstrated that MDP signaling via NOD2 has downregulatory effects on TLR2 signaling, particularly the pathway leading to the induction of IL-12 secretion $(1,2,11)$. Now we show that MDP signaling via NOD2 has, somewhat surprisingly, much more global inhibitory effects in that it inhibits not only TLR2 and IL-12, but also other TLR responses and other cytokine responses. This conclusion is based on in vivo studies that showed that administration of MDP to mice led to the amelioration of both DSS colitis and the hapten (TNBS) colitis and that, furthermore, such amelioration was the result of a direct downregulatory effect on multiple TLR pathways responsible for the production of key inflammatory cytokines such as IL-12 and IL-6. It was also based on in vitro studies that showed first that NOD2 (MDP) prestimulation of human monocyte-derived DCs is followed by a greatly diminished capacity of TLR2, TLR3, TLR4, TLR5, and TLR9 ligands to induce production of IL-12, IL-6, and CXCL10 and second that NOD2 prestimulation of human DCs abolishes the subsequent ability of MDP to synergize with ligands of TLR3 and TLR9 in the induction of IL-12, IL-6, and TNF. That these "tolerogenic" effects of MDP prestimulation were in fact mediated by NOD2 was shown in parallel studies of murine BMDCs, which showed that NOD2 prestimulation of NOD2-deficient cells did not generate an inhibitory effect, whereas prestimulation of NOD2-intact cells did generate such an effect. Overall, these data provide strong evidence that NOD2 prestimulation of APCs negatively regulates inflammatory responses induced by a broad range of TLR ligands and therefore is another explanation of why defective NOD2 function contributes to the pathogenesis of Crohn disease.

The concept that a major function of NOD2 signaling is the regulation of TLR responses had its inception mainly in in vitro studies showing that splenic macrophages or DCs from NOD2deficient mice produce increased amounts of IL-12p40 and -p70 compared with cells from wild-type mice; in addition, responses of cells from NOD2-deficient mice but not wild-type mice could be downregulated with MDP (11). However, these data were questioned by other investigators who also postulated that NOD2 
abnormalities are associated with Crohn disease due to effects of the abnormalities on other, nonregulatory activities of NOD2, including reduced epithelial $\alpha$-defensin production or the overproduction of IL-1 $\beta(30,31)$. In addition, a number of studies have appeared that show that NOD2 activation enhances concomitant TLR stimulation $(32,33)$. Our approach to bridging this discrepancy has been to obtain new and independent data that support the concept of NOD2 regulation. In one such study, we showed that NOD2-deficient mice that received adoptively transferred TCR-transgenic T cells that recognize OVA peptide develop a Th1mediated colitis when exposed to recombinant E. coli expressing OVA (ECOVA); moreover, this in vivo evidence of increased responsiveness of NOD2-deficient mice was abolished in mice that were deficient in both NOD2 and TLR2, indicating that, in this case, NOD2 was mainly regulating an inflammatory response mediated by a particular TLR (18). In another such study, we showed that, whereas mice that are deficient in NOD2 exhibit increased TLR2 responses, mice that overexpress NOD2 as the result of the presence of either a NOD2 transgene or administration of a NOD2expressing plasmid exhibit decreased TLR2 responses; moreover, such mice are less susceptible to 2 forms of induced colitis (21). Interestingly, overexpression of a NOD2 plasmid reflecting the LRR frameshift defect found in Crohn disease was far less effective in protecting mice from the development of colitis. Finally, the studies presented here add increased validity to the concept that NOD2 has downregulatory activity. In the first place, the fact that in vivo administration of MDP to mice prevents colitis in 2 independent murine models of colitis provides rather unequivocal evidence that the net effect of NOD2 signaling (when enhanced by exogenous ligand) is the profound inhibition of TLR responses necessary for inflammation. In the second place, our results show that in vitro responses to MDP (even those that are initially enhancing to concomitant TLR responses) are almost uniformly inhibitory to subsequent TLR responses when NOD2 signaling is provided prior to the time of TLR signaling and an inhibitory substance (IRF4) has had time to accumulate. This last observation helps resolve discrepancies with previous studies showing NOD2 signaling enhancement $(32,33)$.

In our studies of the mechanism of the inhibitory effect of NOD2 prestimulation, we showed that a previously identified TLR inhibitory molecule, IRF4 $(19,20)$, was induced by MDP signaling. Several subsequent studies strongly suggested that IRF4 was, in fact, the mediator of NOD2 inhibition. First, silencing of IRF4 gene expression by either a mixture of or 2 individual IRF4-specific siRNAs abolished the inhibitory effect of NOD2 prestimulation on the human DC responses to TLR2 and TLR4 ligands. Second, neither NOD2 prestimulation of the human monocytic cell line THP1 nor prestimulation of HT-29 epithelial cells inhibited subsequent cytokine responses to TLR ligands, consistent with the finding that both of these cells do not express IRF4. Third and most importantly, the protective effect of MDP administration on TNBS colitis was abolished in mice administered intrarectal IRF4specific siRNA, and the protective effect of MDP on DSS colitis was abolished in IRF4-deficient mice. These in vitro and in vivo studies together provided strong evidence that IRF4 was indeed the major mediator of NOD2 inhibition.

As to the molecular interactions that account for IRF4 inhibition, one has to consider first the report by Negishi et al. in which evidence was presented showing that IRF4 competes with IRF5 for binding to MyD88 and thereby prevents a key step in the MyD88 signaling pathway (19). However, this proposed mechanism of IRF4 inhibition cannot be the sole explanation of the NOD2 inhibitory effect in the studies reported here because the latter inhibitory effect also involves suppression of a TLR pathway not involving MyD88, the TLR3 pathway. An alternative (or additional) mechanism of IRF4 inhibition arises from our cotransfection studies in which various signaling components were overexpressed, IRF4 bound to TRAF6 and RICK as well as MyD88, and such binding resulted in reduction of MyD88, TRAF6, and RICK-mediated NF- $\kappa \mathrm{B}$ activation (Figure 8D). These data lead to the view that the mechanism of IRF4 inhibition is due to its capacity to bind to TRAF6 and/or RICK and thus interfere with the ability of these components to activate the NF- $\mathrm{KB}$ pathway. However, this mechanism is still not completely adequate because it does not explain why inhibition associated with LPS prestimulation is not affected by IRF4 gene silencing despite the fact that LPS prestimulation also induces increased IRF4 expression; in addition, it does not explain why NOD1 prestimulation does not result in inhibition even though such stimulation induces IRF4 expression. Since generation of activated RICK is a prominent if not unique feature of the NOD signaling pathway, one way of resolving this discrepancy is to assume that IRF4 induced by NOD2 prestimulation binds to RICK and the complex formed has a heightened propensity to inhibit TLR signaling, perhaps by interfering with the signaling functions of other TLR components involved in both the MyD88 and TRIF pathways. The fact that NOD2 prestimulation of DCs did lead to the formation of IRF4/RICK complexes under physiologic conditions offers initial support for this idea, but much further work will be necessary before it can be accepted. In particular, it will be important to define far more completely how IRF4 binding to RICK (and perhaps other TLR signaling components as well) affects the function of the TLR signaling pathway.

As alluded to above, we previously reported that MDP, when present in cultures with PGN, downregulates PGN-mediated (TLR2-mediated) NF- $\kappa \mathrm{B}$ activation and thus, that in the absence of NOD2, PGN induces greatly increased IL-12 responses (11). In this case, the effects of MDP on induction of IL-12 appeared to be focused solely on TLR2 responses rather than on all TLR responses, as is the case when cells are prestimulated by MDP. Despite this discrepancy, the inhibitory effect of NOD2 signaling on TLR2 responses in cultures stimulated by MDP and PGN simultaneously is most likely mediated by essentially the same mechanism as that discussed governing the inhibitory effect of NOD2 prestimulation. The data concerning the TLR specificity of the inhibition can be reconciled if we assume that in cultures in which cells are stimulated by TLR ligand and MDP simultaneously, IRF4 levels are not sufficiently high at the time of initial TLR stimulation to suppress all TLR responses but are sufficiently high to suppress TLR 2 responses; in contrast, when cells are stimulated by MDP and TLR ligands sequentially, as in the present studies, sufficient IRF4 is generated by the time of stimulation to affect all responses. The finding that TLR2 responses were in fact more sensitive to IRF4 inhibition than other TLR responses supports this view (Supplemental Figure 7). This ability of NOD2 signaling to exert differential effects depending on the strength or duration of the signaling suggests that such signaling affects mucosal responses at 2 levels. At one level, that occurring with the simultaneous activation of TLRs and NOD2, MDP stimulation results in suppression of the TLR2 pathway and its resulting IL-12 production while leaving intact or even enhancing TNF or IL-8 production by sev- 
eral other TLR pathways (11). Thus, at this level, NOD2 signaling inhibits a potentially inflammatory Th1 response while still allowing (or even facilitating) the development of some elements of host defense. At another level, that occurring when NOD2 signaling occurs prior to TLR signaling or with continuous administration of MDP, one sees inhibition of multiple TLR responses. This level may be operative under physiologic conditions wherein the mucosal system is constantly exposed to commensal flora. It therefore may be the level responsible for the immune dysregulation associated with the CARD15 mutations of Crohn disease.

The state of unresponsiveness induced by preexposure to MDP via NOD2 signaling is not unlike that induced by preexposure to TLR ligands such as LPS and CPG via their respective TLRs, although in vitro studies show that the unresponsiveness induced by each of these stimuli differs both qualitatively and quantitatively from that observed with MDP (T. Watanabe and W. Strober, unpublished observations). This undoubtedly reflects the fact that somewhat different mechanisms underlie the various unresponsive states induced by different ligands. For instance, there is evidence that the state of unresponsiveness induced by LPS is mediated, at least in part, by IRAK-M (23) and not, as in the case of the unresponsiveness induced by MDP, by IRF4 (19). The latter is supported by the fact that in the present study, gene silencing of IRF4 did not affect LPS-induced unresponsiveness and in previous studies by the fact that IRF4-deficient mice manifest normal LPS-induced unresponsiveness (20). In a like manner, the unresponsiveness induced by $\mathrm{CpG}$ is probably due to yet a third mechanism, since in a recent study it was shown that this unresponsiveness is dependent on intact TLR9 signaling and induction of type I IFN (34). Finally, it should be noted that the ability of NOD2 signaling to limit TLR responses does not conflict with recent data showing that signaling via TLR ligands can ameliorate mucosal inflammation under circumstances in which exogenous ligand is administered $(34,35)$. This follows from the fact that, while TLR signaling is essential for gut inflammation (36), the latter can, in principle, be regulated by TLR signaling or NOD2 signaling, particularly when these are induced by the administration of exogenous ligands under conditions that favor the buildup of inhibitory factors.

In conclusion, it is important to mention that these findings have certain implications both with respect to the possible mechanism by which CARD15 mutations function as susceptibility factors in Crohn disease and with respect to a possible new approach to treatment of this disease. As shown in the study of NOD2-deficient mice reconstituted with plasmids expressing a NOD2 construct with a CARD15 frame-shift mutation, the NOD2 resulting from a CARD15 mutation does not confer MDP-mediated protection from DSS colitis. These data, and by implication, the data obtained in NOD2-intact mice imply that in patients with Crohn disease-related CARD15 mutations, an important negative regulatory mechanism with respect to responses to the various TLR ligands associated with the normal mucosal microflora is impaired and the background innate immune response potentially induced by these ligands is correspondingly enhanced. While this in itself may not be sufficient to induce pathologic inflammation of the gastrointestinal tract, it could set the stage for such inflammation when, as already mentioned, a second abnormality is also present, such as a propensity to mount an adaptive immune response to one or another antigen associated with the intestinal microflora (18). With respect to treatment, the present findings showing that NOD2-ligand (MDP) administration to normal mice inhibits or abolishes induced experimental colitis implies that MDP administration to Crohn disease patients without NOD2 mutations may prevent recurrence of disease. It should be noted, however, that this potential therapy must first contend with the fact that MDP or certain derivatives of MDP have been shown to have adjuvant properties possibly because, as noted here and previously, activation of NOD2 can transiently enhance certain immune responses before it exerts a more dominant inhibitory response (37-39); in addition, MDP administration to mice has been noted to have arthritogenic properties (40). The therapeutic use of MDP is nevertheless still possible because not all MDP preparations have these effects and the latter may be dependent on dose or route of administration. Thus, while the use of MDP for the treatment of Crohn disease must proceed with caution, it remains a viable possibility.

\section{Methods}

Reagents. Recombinant human and murine GM-CSF and IL-4 were from Peprotech. NOD2 ligand MDP was from Sigma-Aldrich. NOD1 ligand $(\gamma$ DGDAP) was synthesized by the Peptide Institute. Unless otherwise described, the doses of TLR ligands and NOD ligands used for stimulation were as follows: PGN (TLR2 ligand, $10 \mu \mathrm{g} / \mathrm{ml}$; Fluka); LTA (lipoteichoic acid, TLR2 ligand, $10 \mu \mathrm{g} / \mathrm{ml}$ ); Pam ${ }_{3} \mathrm{CSK} 4$ (Pam, TLR2 ligand, $1 \mu \mathrm{g} / \mathrm{ml}$; InvivoGen); dsRNA (TLR3 ligand, $50 \mu \mathrm{g} / \mathrm{ml}$; InvivoGen); LPS (TLR4 ligand, $1 \mu \mathrm{g} / \mathrm{ml}$; Sigma-Aldrich); flagellin (TLR5 ligand, $1 \mu \mathrm{g} / \mathrm{ml}$; InvivoGen); and CPG (TLR9 ligand, $1 \mu \mathrm{M}$; InvivoGen); MDP (NOD2 ligand, $10 \mu \mathrm{g} / \mathrm{ml}$ ); and $\gamma$ DGDAP (NOD1 ligand, $10 \mu \mathrm{g} / \mathrm{ml}$ ).

Induction of colitis. TNBS colitis was induced in C57BL/10 mice obtained from Jackson Laboratories, as described previously (41). On days $-3,-2$, and -1 , mice received i.p. injection of MDP $(100 \mu \mathrm{g})$ or PBS for a total of 3 times before intrarectal administration of $3.75 \mathrm{mg}$ of TNBS in $100 \mu \mathrm{l}$ of $45 \%$ ethanol. MLN cells and colon LP lymphocytes were isolated on day 3 , as described previously (42). Cells $\left(1 \times 10^{6} / \mathrm{ml}\right)$ were stimulated with anti-CD3 $(1 \mu \mathrm{g} / \mathrm{ml}$; BD Biosciences - Pharmingen $)$ and TLR ligands, as described above. In the case of LP lymphocytes, cells were stimulated with TLR ligands in the presence of murine IFN- $\gamma(20 \mathrm{ng} / \mathrm{ml}$; Peprotech). Cultured supernatants were collected at 24 hours and analyzed for cytokine production by ELISA. In some experiments, whole-cell extracts were prepared from splenic and MLN CD $11 \mathrm{~b}^{+}$cells from mice on day 0 for the analysis of IRF4 expression. CD11 $\mathrm{b}^{+}$cells were isolated as described previously (11). For the induction of DSS colitis experiments, NOD2-intact, NOD2deficient, or IRF4-deficient mice (29) were given a range of DSS doses that included $4 \%, 5 \%$, or $5.5 \%$ DSS (MW 36,000-50,000; ICN Biomedicals) in drinking water for 6 days (days $0-5$ ) and then placed on regular water for 2 days (days 6-7). Mice were administered MDP (100 $\mu \mathrm{g}$ i.p.) or PBS for a total of 3 times on days 0,1 , and 2 . MLN cells $\left(1 \times 10^{6} / \mathrm{ml}\right)$ were stimulated with TLR ligands, as described above. In the experiments in which mice received intact-NOD2, frameshift-NOD2, or control-empty vector plasmid, plasmids were encapsulated in HVJ (GenomIdea) using protamine sulfate according to the manufacturer's protocol. $100 \mu \mathrm{g} / \mathrm{mouse}$ of encapsulated plasmid with $100 \mu \mathrm{g} /$ mouse of MDP were administered i.p. on days 0,1 , and 2. Animal use was approved by the NIH Animal Care and Use Committee. All animal use adhered to NIH Animal Care Guidelines.

Plasmids encoding intact NOD2 and frameshift NOD2. A pcDNA4HisMax plasmid encoding mouse NOD2 complementary DNA was used as intact NOD2. Mutated NOD2 plasmid was obtained by inserting a mutation into intact NOD2 by the primer 5'-CAGAAGCCCTCCTGCAGGCCCCTTAAGGGAACAGTGCCATTCTGGAG-3' and its antisense primer using the QuikChange II XL Site-Directed Mutagenesis Kit (Stratagene). This mouse NOD2 cDNA is equivalent to the human 3020ins C Crohn disease frameshift mutation. This mutated NOD2 will be referred to as frameshift NOD2. 
Human monocyte-derived DCs. Monocytes were elutriated from the peripheral blood of healthy donors and were cultured in 6-well plates $\left(1 \times 10^{6} / \mathrm{ml}\right)$ in $5 \mathrm{ml}$ of complete medium (RPMI 1640 medium supplemented with $2 \mathrm{mM}$ L-glutamine and $10 \%$ fetal calf serum) supplemented with recombinant GM-CSF $(20 \mathrm{ng} / \mathrm{ml})$ and recombinant IL-4 $(20 \mathrm{ng} / \mathrm{ml})$. After 3 days of culture, half of the medium in each well was exchanged. After 6 days of culture, more than $90 \%$ of the cells expressed characteristic DC-specific markers (CD1a and HLA-DR), as determined by flow cytometry. After being washed twice, cells $\left(1 \times 10^{6} / \mathrm{ml}\right)$ were incubated with MDP, LPS, CPG, or medium for 24 hours in the absence of GM-CSF and IL-4 and then stimulated with a broad range of TLR ligands in the presence or absence of MDP after washing 3 times. Cultured supernatants were collected at 24 hours and analyzed for cytokine and chemokine production by ELISA. In some experiments, DCs $\left(2 \times 10^{6} / \mathrm{ml}\right)$ were transfected with $2 \mu \mathrm{g}$ of control siRNA, IRF4 siRNA, or IRAK-M siRNA (Santa Cruz Biotechnology Inc.) by a human DC nucleofection kit (Amaxa). DCs were transfected with either a mixture of or 2 individual IRF4-specific siRNAs. The sequences of IRF4 siRNA were as follows: IRF4 siRNA no. 1, 5'-CUCCUUUCCUAUCUUUACAUU-3'; IRF4 siRNA no. 2, 5'-GGUAGGUAUUAGUGUUUGAUU-3'. siRNA-transfected DCs were treated with MDP or LPS followed by stimulation with TLR ligands, as described above.

Murine BMDCs. BM cells were prepared from NOD2-intact and NOD2deficient mice (43) and cultured in 6-well plates $\left(1 \times 10^{6} / \mathrm{ml}\right)$ in $5 \mathrm{ml}$ of complete RPMI medium supplemented with recombinant GM-CSF (20 $\mathrm{ng} / \mathrm{ml})$ and recombinant IL-4 $(20 \mathrm{ng} / \mathrm{ml})$. After 3 days of culture, half of the medium in each well was exchanged. On day 6 , cells were harvested and sorted by anti-mouse CD11c magnetic beads (Miltenyi Biotech), as described previously (44). CD11 $\mathrm{c}^{+} \mathrm{DCs}\left(1 \times 10^{6} / \mathrm{ml}\right)$ were incubated with MDP $(50 \mu \mathrm{g} / \mathrm{ml})$ or medium for 24 hours and then stimulated with a broad range of TLR ligands, as described above. Cultured supernatants were collected at 48 hours and analyzed for cytokine production by ELISA. In some experiments, CD $11 \mathrm{c}^{+}$DCs $\left(2 \times 10^{6} / \mathrm{ml}\right)$ preincubated with MDP or medium were cocultured with splenic CD4+ $\mathrm{T}$ cells $\left(1 \times 10^{6} / \mathrm{ml}\right)$ isolated from OT-II transgenic mice (Jackson Laboratories) (45) in the presence of $\mathrm{OVA}_{323-339}$ peptide $(0.5 \mu \mathrm{M})$ and TLR ligands, as described above. Splenic $\mathrm{CD} 4^{+} \mathrm{T}$ cells were purified with the use of anti-mouse CD4 magnetic beads (Miltenyi Biotech), as described previously (46). Cultured supernatants were collected at 60 hours and analyzed for cytokine production by ELISA. In the experiments staining for surface costimulatory molecules, CD11 $\mathrm{c}^{+}$DCs from NOD2-intact mice were incubated with $\operatorname{MDP}(50 \mu \mathrm{g} / \mathrm{ml})$ or medium for 24 hours and then stained with antiCD80, anti-CD86 (eBioscience), and anti-MHC class II (Miltenyi Biotech) followed by flow cytometric analysis.

Immunoprecipitation. HEK293 cells (ATCC) $\left(1 \times 10^{6} /\right.$ cells) were transfected with $2 \mu \mathrm{g}$ of FLAG-tagged human IRF4 vector together with $2 \mu \mathrm{g}$ of human MyD88, TRAF6, RICK, NOD2 vector (InvivoGen), or TRAF2 vector (Origene) by Trans IT LT1 (Mirus). Whole-cell lysates were prepared 48 hours after the transfection and were incubated with anti-FLAG-conjugated beads (Sigma-Aldrich) overnight. In some experiments, human DCs were incubated with anti-IRF4 Abs (Santa Cruz Biotechnology Inc.) and protein $\mathrm{A} / \mathrm{G}$ plus agarose (Santa Cruz Biotechnology Inc.).

Luciferase assay. HT-29 cells $\left(1 \times 10^{6} / \mathrm{ml}\right.$; ATCC $)$ were transiently transfected

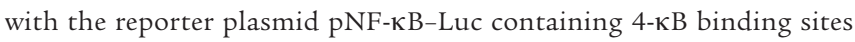
(Clontech) and pSV- $\beta$-galactosidase vector (Promega) together with a plasmid expressing MyD88, TRAF6, RICK, and IRF4 by Trans-IT LT1 reagent. After overnight incubation with serum-free medium, cell lysates were analyzed for luciferase activity (Promega) and galactosidase activity (Applied Biosystems) for normalization. The luciferase activity was normalized for transfection efficiency and is called $N F-\kappa B$ activation fold in the text.
Human monocytic cell lines. Two human monocytic cell lines, THP1 and Monomac6, were used. Cells $\left(5 \times 10^{5} / \mathrm{ml}\right)$ were preincubated with MDP, LPS, or medium for 24 hours and stimulated with TLR ligands after washing 3 times. Cultured supernatants were collected at 24 hours and analyzed for cytokine production by ELISA. In some experiments, THP1 and human DCs were transfected with a vector expressing human IRF4 cDNA or a control vector by Amaxa followed by stimulation with MDP and TLR ligands.

IRF4 siRNA study in TNBS colitis. Sequences of siRNAs were as follows: control siRNA, 5'-UAAGGCUAUGAAGAGAUACUU-3'; and IRF4 siRNA, 5'-GGACACACCUAUGAUGUUAUU-3'. These siRNAs were obtained from Dharmacon. For in vivo transfection, $1 \mathrm{mg}$ siRNA was encapsulated in HVJ as previously described $(11,41)$. C57BL/10 mice that were treated with MDP during days -3 and -1 were intrarectally administered $100 \mu \mathrm{g}$ of IRF 4 or control siRNA encapsulated in HVJ envelope for a total of 4 times on days $-2,-1,0$, and 1 .

Histological analysis. Colon was harvested at the indicated time points. Colon tissues were stained with $\mathrm{H} \& \mathrm{E}$ and used for the scoring of inflammation, as described by Obermeier et al. for DSS colitis (47) and Neurath et al. for TNBS colitis (48). Histology was scored for DSS colitis as follows for epithelium: 0 , normal morphology; 1 , loss of goblet cells; 2 , loss of goblet cells in large areas; 3 , loss of crypts; and 4 , loss of crypts in large areas. For infiltration, scoring was as follows: 0 , no infiltrate; 1 , infiltrate around crypt basis; 2 , infiltrate reaching to lamina muscularis mucosa; 3 , extensive infiltration reaching the lamina muscularis mucosae and thickening of the mucosa with abundant edema; and 4, infiltration of the lamina submucosa. The total histological score is given as epithelium plus infiltration. Histology was scored for TNBS colitis as follows: 0 , no sign of inflammation; 1 , very low level; 2 , low level of leukocytic infiltration; 3 , high level of leukocytic infiltration, high vascular density, and thickening of the colon wall; and 4, transmural infiltrations, loss of goblet cells, high vascular density, and thickening of the colon wall.

ELISA. Protein concentrations of cytokines and chemokines were determined by BD Biosciences - Pharmingen ELISA kits for assay of human IL-12p40, human IL-12p70, human TNF, human IL-10, human CXCL8, human CXCL10, mouse IL-12p40, mouse IL-12p70, mouse IL-6, and mouse IFN- $\gamma$; eBioScience kits for assay of human IL-6; and Biosource kits for assay of mouse serum amyloid A.

Western blot analysis. Cell lysis and blotting was done as described (11). The membrane was blotted with Abs as follows: anti-TAK1, MyD88, TRAF6, IRF3, SOCS1, anti-mouse IRF4 (Santa Cruz Biotechnology Inc.), anti-human IRF4, anti-IRAK-M, anti-IKK- $\gamma$ (Cell Signaling), anti-IRF5 (Abcam), and RICK (Cayman Chemicals).

Gel-shift assays. Nuclear extracts were prepared from human DCs preincubated with MDP or medium for 24 hours and stimulated with TLR ligands for 2 hours. In colitis experiments, MLN cells isolated from mice at the indicated time points were stimulated with TLR ligands for 2 hours, and then nuclear extracts were prepared. Nuclear extracts were obtained with the use of TransFactor Extraction Kit (Clontech). Analysis of NF-kB activation by gel-shift assay was performed according to the protocol contained in the Gel-shift NF-кB kit (Active Motif). Analysis of p65 and c-Rel activation was measured by TransAM Kit (Active Motif).

Statistics. Two-tailed Student's $t$ test was used to evaluate the significance of differences. Statistical analysis was performed with the StatView v.4.5 program (Abacus Concepts). $P<0.05$ was regarded as statistically significant. Results are presented as means \pm SEM unless otherwise described.

\section{Acknowledgments}

We would like to thank K. Honma, K. Yui, and T. Matsuyama for providing us with a plasmid-expressing human IRF4 and murine 
IRF4. This study is supported in part by grants from the Ministry of Education, Science and Culture, Japan, the Japan Society for the Promotion of Science (19590495), and the Shimizu Foundation for Immunology Research.

Received for publication June 29, 2007, and accepted in revised form November 15, 2007.

1. Strober, W., Murray, P.J., Kitani, A., and Watanabe, T. 2006. Signalling pathways and molecular interactions of NOD1 and NOD2. Nat. Rev. Immunol. 6:9-20.

2. Watanabe, T., Kitani, A., and Strober, W. 2005. NOD2 regulation of Toll-like receptor responses and the pathogenesis of Crohn's disease. Gut. 54:1515-1518.

3. Inohara, Chamaillard, McDonald, C., and Nunez, G. 2005. NOD-LRR proteins: role in host-microbial interactions and inflammatory disease. Annu. Rev. Biochem. 74:355-383.

4. Hugot, J.P., et al. 2001. Association of NOD2 leucine-rich repeat variants with susceptibility to Crohn's disease. Nature. 411:599-603.

5. Ogura, Y., et al. 2001. A frameshift mutation in NOD2 associated with susceptibility to Crohn's disease. Nature. 411:603-606.

6. Inohara, N., et al. 2003. Host recognition of bacterial muramyl dipeptide mediated through NOD2. Implications for Crohn's disease. J. Biol. Chem. 278:5509-5512.

7. Girardin, S.E., et al. 2003. Nod2 is a general sensor of peptidoglycan through muramyl dipeptide (MDP) detection. J. Biol. Chem. 278:8869-8872.

8. Marks, D.J., et al. 2006. Defective acute inflammation in Crohn's disease: a clinical investigation. Lancet. 367:668-678.

9. Dziarski, R., and Gupta, D. 2005. Staphylococcus aureus peptidoglycan is a toll-like receptor 2 activator: a reevaluation. Infect. Immun. 73:5212-5216.

10. Akira, S., Takeda, K., and Kaisho, T. 2001. Tolllike receptors: critical proteins linking innate and acquired immunity. Nat. Immunol. 2:675-680.

11. Watanabe, T., Kitani, A., Murray, P.J., and Strober, W. 2004. NOD2 is a negative regulator of Toll-like receptor 2-mediated T helper type 1 responses. Nat. Immunol. 5:800-808.

12. Bouma, G., and Strober, W. 2003. The immunological and genetic basis of inflammatory bowel disease. Nat. Rev. Immunol. 3:521-533.

13. Strober, W., Fuss, I.J., and Blumberg, R.S. 2002. The immunology of mucosal models of inflammation. Annu. Rev. Immunol. 20:495-549.

14. Mannon, P.J., et al. 2004. Anti-interleukin-12 antibody for active Crohn's disease. N. Engl. J. Med. 351:2069-2079.

15. Uehara, A., et al. 2005. Muramyldipeptide and diaminopimelic acid-containing desmuramylpeptides in combination with chemically synthesized Toll-like receptor agonists synergistically induced production of interleukin-8 in a NOD2- and NOD1-dependent manner, respectively, in human monocytic cells in culture. Cell. Microbiol. 7:53-61.

16. Netea, M.G., et al. 2005. Nucleotide-binding oligomerization domain-2 modulates specific TLR pathways for the induction of cytokine release. J. Immunol. 174:6518-6523.

17. van Heel, D.A., et al. 2005. Muramyl dipeptide and toll-like receptor sensitivity in NOD2-associated

Address correspondence to: Warren Strober, National Institute of Allergy and Infectious Diseases, NIH, Building 10-CRC Room 5W3940, 10 Center Drive, Bethesda, Maryland 20892, USA. Phone: (301) 496-6810; Fax: (301) 402-2240; E-mail: wstrober@niaid.nih.gov.

Tomohiro Watanabe and Naoki Asano contributed equally to this work.

Crohn's disease. Lancet. 365:1794-1796.

18. Watanabe, T., et al. 2006. Nucleotide binding oligomerization domain 2 deficiency leads to dysregulated TLR2 signaling and induction of antigen-specific colitis. Immunity. 25:473-485.

19. Negishi, H., et al. 2005. Negative regulation of Tolllike-receptor signaling by IRF-4. Proc. Natl. Acad. Sci. U. S. A. 102:15989-15994.

20. Honma, K., et al. 2005. Interferon regulatory factor 4 negatively regulates the production of proinflammatory cytokines by macrophages in response to LPS. Proc. Natl. Acad. Sci. U. S. A. 102:16001-16006.

21. Yang, Z., et al 2007. NOD2 transgenic mice exhibit enhanced MDP-mediated down-regulation of TLR2 responses and resistance to colitis induction. Gastroenterology. 133:1510-1521.

22. Langenkamp, A., Messi, M., Lanzavecchia, A., and Sallusto, F. 2000. Kinetics of dendritic cell activation: impact on priming of TH1, TH2 and nonpolarized T cells. Nat. Immunol. 1:311-316.

23. Kobayashi, K., et al. 2002. IRAK-M is a negative regulator of Toll-like receptor signaling. Cell. 110:191-202.

24. Gingras, S., Parganas, E., de Pauw, A., Ihle, J.N., and Murray, P.J. 2004. Re-examination of the role of suppressor of cytokine signaling 1 (SOCS1) in the regulation of toll-like receptor signaling. J. Biol. Chem. 279:54702-54707.

25. Lehtonen, A., et al. 2005. Differential expression of IFN regulatory factor 4 gene in human monocytederived dendritic cells and macrophages. J. Immunol. 175:6570-6579.

26. Gutierrez, O., et al. 2002. Induction of Nod2 in myelomonocytic and intestinal epithelial cells via nuclear factor-kappa B activation. J. Biol. Chem. 277:41701-41705.

27. McDonald, C., et al. 2005. A role for Erbin in the regulation of Nod2-dependent NF-kappaB signaling. J. Biol. Chem. 280:40301-40309.

28. Yoshida, K., et al. 2005. Active repression of IFN regulatory factor-1-mediated transactivation by IFN regulatory factor-4. Int. Immunol. 17:1463-1471.

29. Tamura, T., et al. 2005. IFN regulatory factor-4 and 8 govern dendritic cell subset development and their functional diversity. J. Immunol. 174:2573-2581.

30. Kobayashi, K.S., et al. 2005. Nod2-dependent regulation of innate and adaptive immunity in the intestinal tract. Science. 307:731-734.

31. Maeda, S., et al. 2005. Nod2 mutation in Crohn's disease potentiates NF-kappaB activity and IL-1beta processing. Science. 307:734-738.

32. Tada, H., Aiba, S., Shibata, K., Ohteki, T., and Takada, H. 2005. Synergistic effect of Nod1 and Nod2 agonists with toll-like receptor agonists on human dendritic cells to generate interleukin-12 and T helper type 1 cells. Infect. Immun. 73:7967-7976.

33. Uehori, J., et al. 2005. Dendritic cell maturation induced by muramyl dipeptide (MDP) derivatives: monoacylated MDP confers TLR2/TLR4 activation. J. Immunol. 174:7096-7103.
34. Katakura, K., et al. 2005. Toll-like receptor 9induced type I IFN protects mice from experimental colitis. J. Clin. Invest. 115:695-702.

35. Vijay-Kumar, M., et al. 2007. Activation of toll-like receptor 3 protects against DSS-induced acute colitis. Inflamm. Bowel Dis. 13:856-864.

36. Rakoff-Nahoum, S., Hao, L., and Medzhitov, R. 2006. Role of toll-like receptors in spontaneous commensal-dependent colitis. Immunity. 25:319-329.

37. Chedid, L., et al. 1979. Enhancement of certain biological activities of muramyl dipeptide derivatives after conjugation to a multi-poly (DL-alanine)-poly (L-lysine) carrier. Proc. Natl. Acad. Sci. U. S. A. 76:6557-6561.

38. Tsujimoto, M., Kotani, S., Shiba, T., and Kusumoto, S. 1986. Adjuvant activity of 6-O-acyl-muramyldipeptides to enhance primary cellular and humoral immune responses in guinea pigs: dose-response and local reactions observed with selected compounds. Infect. Immun. 53:517-521.

39. Parant, M., et al. 1990. Priming effect of muramyl peptides for induction by lipopolysaccharide of tumor necrosis factor production in mice. J. Lenkoc. Biol. 47:164-169.

40. Koga, T., et al. 1986. Muramyl dipeptide induces acute joint inflammation in the mouse. Microbiol. Immunol. 30:717-723.

41. Fichtner-Feigl, S., Fuss, I.J., Preiss, J.C., Strober, W., and Kitani, A. 2005. Treatment of murine Th1- and Th2-mediated inflammatory bowel disease with NF-kappa B decoy oligonucleotides. J. Clin. Invest. 115:3057-3071.

42. Watanabe, T., Yamori, M., Kita, T., Chiba, T., and Wakatsuki, Y. 2005. CD4+CD25+ T cells regulate colonic localization of CD4 T cells reactive to a microbial antigen. Inflamm. Bowel Dis. 11:541-550.

43. Pauleau, A.L., and Murray, P.J. 2003. Role of nod2 in the response of macrophages to toll-like receptor agonists. Mol. Cell. Biol. 23:7531-7539.

44. Watanabe, T., et al. 2003. A liver tolerates a portal antigen by generating $\mathrm{CD} 11 \mathrm{c}+$ cells, which select Fas ligand + Th2 cells via apoptosis. Hepatology. 38:403-412.

45. Kondrack, R.M., et al. 2003. Interleukin 7 regulates the survival and generation of memory CD 4 cells. J. Exp. Med. 198:1797-1806.

46. Watanabe, T., et al. 2002. Administration of an antigen at a high dose generates regulatory CD4+ $\mathrm{T}$ cells expressing CD95 ligand and secreting IL-4 in the liver. J. Immunol. 168:2188-2199.

47. Obermeier, F., et al. 1999. Interferon-gamma (IFN-gamma)- and tumour necrosis factor (TNF)induced nitric oxide as toxic effector molecule in chronic dextran sulphate sodium (DSS)-induced colitis in mice. Clin. Exp. Immunol. 116:238-245.

48. Neurath, M.F., Fuss, I., Kelsall, B.L., Stuber, E., and Strober, W. 1995. Antibodies to interleukin 12 abrogate established experimental colitis in mice. J. Exp. Med. 182:1281-1290. 